\title{
MoMuLV-ts-1: A Unique Mouse Model of Retrovirus-Induced Lymphoma Transmitted by Breast Milk
}

\author{
J. Chakraborty, ${ }^{1}$ H. Okonta, ${ }^{1,2}$ H. Bagalb, ${ }^{1}$ and J. Duggan ${ }^{1,2}$ \\ ${ }^{1}$ Department of Physiology and Pharmacology, Block Health Science Building, College of Medicine, University of Toledo, \\ Health Science Campus, Toledo, $\mathrm{OH} 43614$, USA \\ ${ }^{2}$ Department of Medicine, Ruppert Building, College of Medicine, University of Toledo, Health Science Campus, \\ Toledo, OH 43614, USA \\ Correspondence should be addressed to J. Chakraborty, joana.chakraborty@utoledo.edu
}

Received 31 January 2011; Revised 16 May 2011; Accepted 6 June 2011

Academic Editor: Paul Jolicoeur

Copyright (๑) $2011 \mathrm{~J}$. Chakraborty et al. This is an open access article distributed under the Creative Commons Attribution License, which permits unrestricted use, distribution, and reproduction in any medium, provided the original work is properly cited.

Our laboratory has developed a murine model of lymphoma via breast milk transmission of MoMuLV-ts-1 (Moloney murine leukemia virus-temperature sensitive mutant-1). Uninfected offspring suckled from infected surrogate mothers become infected and develop lymphoma. Multiple gene integration sites of ts-1 into the infected mouse genome including tacc3, aurka, ndel1, tpx2, p53, and rhamm were identified, and mRNA expressions were quantitated. These genes produce centrosomal proteins, which may be involved in abnormal chromosomal segregation leading to aneuploidy or multiploidy, thus causing lymphoma. Since there is no report to date on this retroviral model leading to centrosomal abnormality, and causing lymphoma development, this is a valuable and unique model to study the centrosomal involvement in lymphomagenesis.

\section{Introduction}

Retroviral infections in humans range from asymptomatic murine leukemia virus infections to deadly infections such as HIV. The HIV epidemic has spurred unprecedented levels of retroviral research in the last three decades due to the everincreasing human toll. According to the WHO and UNAIDS report in 2008 [1], globally 33.4 million people are now living with HIV. Of these, 2.1 million children under the age of 15 years are suffering from HIV infection and 280,000 children died of AIDS and AIDS-related conditions such as lymphoma in 2008. In 2008, an additional 430,000 children became infected, with the vast majority living in developing countries with little access to antiretroviral therapy (ART). The main route of infection in these children is mother to child transmission (MTCT). Although treatment of infected mothers during pregnancy with ART and use of infant formula after delivery has limited MTCT of HIV-1 in developed countries [1], these options are often unavailable in Africa and other developing countries where MTCT still remains a significant source of HIV infection.
Due to practical and ethical constraints involving human subjects, the mechanism of perinatal transmission of HIV1 is not yet fully understood. HIV can be transmitted to the fetus/infant during prenatal and postnatal periods. A suitable animal model may allow in depth study of the pathophysiologic mechanisms of MTCT of HIV. We have successfully developed a murine model for mother-tooffspring transmission of Moloney Murine Leukemia virus (MoMuLV) ts-1, a retrovirus which mimics HIV. We have clearly demonstrated that the transmission of ts- 1 can occur in utero, intrapartum, and/or postpartum. Transmission of ts- 1 produces an immunodeficiency state with wasting, increased infection, and neurologic deterioration. In addition, postpartum transmission of ts- 1 is associated with an increased development of lymphoma in pups $[2,3]$.

Chakraborty et al. [4] have found that ts-1 integration leads to an overexpression of four genes associated with lymphoma in BALB/c mice infected by breast milk. This unique model has allowed us to demonstrate ts- 1 retrovirus transmission via breast milk and study the molecular mechanism of the lymphoma development through natural 
TABle 1: Comparison between HIV and MoMuLV-ts-1 showing the similarities and differences between these two types of viruses.

\begin{tabular}{lcc}
\hline Characteristics & HIV & ts-1 \\
\hline Retrovirus & + & + \\
Lentivirus & + & $-{ }^{*}$ \\
Initial target for infection: CD4 cells & + & + \\
Depletion of T-lymphocytes & + & + \\
T-cell death via apoptosis & + & + \\
Inefficient transport of env precursor & + & + \\
Viral induced cytokine production & + & + \\
Polyclonal activation of B lymphocytes & + & + \\
Infection of astrocytes and microglia & + & + \\
Demyelination & + & + \\
Neuronal death & + & + \\
Wasting & + & + \\
Can cause lymphoma in some cases*** & + & + \\
Horizontal transmission & + & $-{ }^{* *}$ \\
Mother-to-offspring transmission & + & + \\
Can cause disease & New born & New born \\
* MoMuLV-ts-1: mammalian gamma oncoretrovirus. & ${ }^{* *}$ ts-1: No adult \\
mouse can be infected; therefore, no sexual transmission occurs. & $* * *$ Both \\
HIV and ts-1 may indirectly cause lymphoma. & &
\end{tabular}

transmission of a retrovirus to the offspring. Our hypothesis is that viral integration into the host genome alters host gene expression leading to an abnormal mRNA expression and abnormal protein production. These abnormal proteins alter the centrosome function during the cell cycle resulting in lymphomagenesis. This paper will explore two related areas of ts- 1 research: ts- 1 as a small animal model of perinatal retroviral transmission and ts- 1 in lymphomagenesis.

\section{Part I. MoMuLV-ts-1 as a Small Animal Model}

2.1. The Similarities and Differences between HIV and $M o M u L V$-ts-1. HIV shares many characteristics with ts1. These include CD4 cell targeting, secondary infections, neurodegenerative diseases, macrophage and CD4 cell infection, immunodeficiency, neurotropism, CD4 cell depletion, wasting, lymphomas, and perinatal transmission. However, the mechanism of entry of these two viruses are different. The Moloney murine leukemia virus (MoMuLV) ts- 1 is a temperature sensitive mutant virus [5-7] first isolated by propagating MoMuLV in a thymus bone marrow cell line (TB) taken from CFW/D mice. This virus has a defect in the intracellular processing of the envelope precursor polyprotein (Pr80env) at the restrictive temperature [6-9]. Like HIV, MoMuLV ts- 1 infects CD4 cells, with subsequent CD4 depletion and a resulting immunodeficiency (see Table 1) [10]. ts- 1 is a murine gamma retrovirus that can induce Tcell lymphomas in susceptible strains of mice after a long incubation period [11].

As a simple retrovirus, ts- 1 has only three genes (gag, env, and pol). Infection with ts-1 results in an AIDS-like syndrome in mice similar to HIV infection in humans $[10,12-14]$. The most important characteristic of ts1 to our study is its effects on MTCT by breastfeeding in mice [2-4]. About $97 \%$ of uninfected neonatal mice that suckle from an infected mother develop clinically symptomatic ts1 infection $[3,4]$. Newborn BALB/c mice infected with ts1 virus suffer from neurodegenerative disease resulting in hind limb paralysis and immunologic disease characterized by severe thymic atrophy associated with immunodeficiency due to destruction of T-lymphocytes, and generalized body wasting $[7,15]$. Infectivity of ts- 1 is significantly related to its temperature sensitivity [7], and can replicate optimally at a permissive temperature of $34^{\circ} \mathrm{C}$ [16]. This may explain why the ts- 1 virus can produce hind limb paralysis in newborn mice and not in the adult, because the body temperature of the newborn mice is lower $\left(\sim 34^{\circ} \mathrm{C}\right)$ than that of adult $\left(38.4^{\circ} \mathrm{C}\right)$ [17]. The uniqueness of the ts- 1 among other murine retroviruses is that it can cause degenerative diseases in mice similar to HIV in humans, by affecting both the central nervous system (CNS) and the immune system. Infected T-lymphocytes have impaired function $[14,18]$.

The murine ts- 1 model has been extensively used as a small-animal model for retrovirus-induced neurodegenerative disease [19]. Oxidative stress has been suggested as a major mechanism for ts1-induced neurodegeneration and Tcell loss in infected newborn mice [20]. The U3 region of ts-1 controls the pathogenicity and targets cell type [21].

Transfer of humoral immunity to ts- 1 can be passed from mother to baby via breast milk and can provide protection from neurodegenerative and immunologic disease in neonatal mice [22]. Chakraborty et al. [23], have further developed this murine model to study the perinatal transmission of ts- 1 . Infected mothers can transmit the ts- 1 virus vertically to offspring. They further demonstrated that mother-to-offspring transmission via breast milk can occur at nearly a $100 \%$ incidence [4], and can cause lymphoma when control pups suckle from ts- 1 infected mothers. The pattern of proviral ts1 integration sites observed in these lymphoma tissues correlates with the upregulation of mRNA expression of candidate genes that may contribute to lymphomagenesis [4].

2.2. Mother-to-Child Transmission of HIV by Breastfeeding. MTCT of HIV can occur in utero, intrapartum, or through breastfeeding. Current strategies aimed at decreasing MTCT of HIV have focused mainly on in utero and intrapartum transmission. Prophylaxis with highly active antiretroviral therapy (HAART) during pregnancy and through delivery has substantially reduced the rate of MTCT of HIV at birth [24]. Avoidance of breastfeeding by HIV-infected mothers is the norm in developed countries throughout the world [25]. However, for socioeconomic and cultural reasons, such avoidance is not an acceptable or viable alternative for many HIV-infected women in Africa and other resource poor areas worldwide [26]. In these areas, breastfeeding is the best and only source of infant nutrition, and safe alternatives do not exist because of inadequate supplies of formula and the lack of clean water. Breastfeeding, therefore, accounts for a substantial proportion of the overall MTCT 
TABle 2: Percentages of changes in spleen, lymph node, and thymus due to MTCT of ts-1 virus by breastfeeding in control and infected pups.

\begin{tabular}{|c|c|c|c|c|c|}
\hline Group & Description & Sample size $(n)$ & Splenomegaly & $\begin{array}{l}\text { Lymph node } \\
\text { hypertrophy }\end{array}$ & $\begin{array}{c}\text { Thymic } \\
\text { hypertrophy }\end{array}$ \\
\hline 1 & Control pup nursed by infected surrogate mother & 93 & $95.7 \%$ & $79.1 \%$ & $95.7 \%$ \\
\hline 2 & Infected pup nursed by infected surrogate mother & 94 & $92.6 \%$ & $56.5 \%$ & $92.6 \%$ \\
\hline 3 & Infected pup nursed by control surrogate mother & 108 & $46.3 \%$ & $25.9 \%$ & $46.3 \%$ \\
\hline 5 & Infected pup nursed by biological mother & 88 & $89.8 \%$ & $61.4 \%$ & $89.8 \%$ \\
\hline $4 \& 6$ & Control—no infection & 154 & $1.9 \%$ & $0.0 \%$ & $5.8 \%$ \\
\hline
\end{tabular}

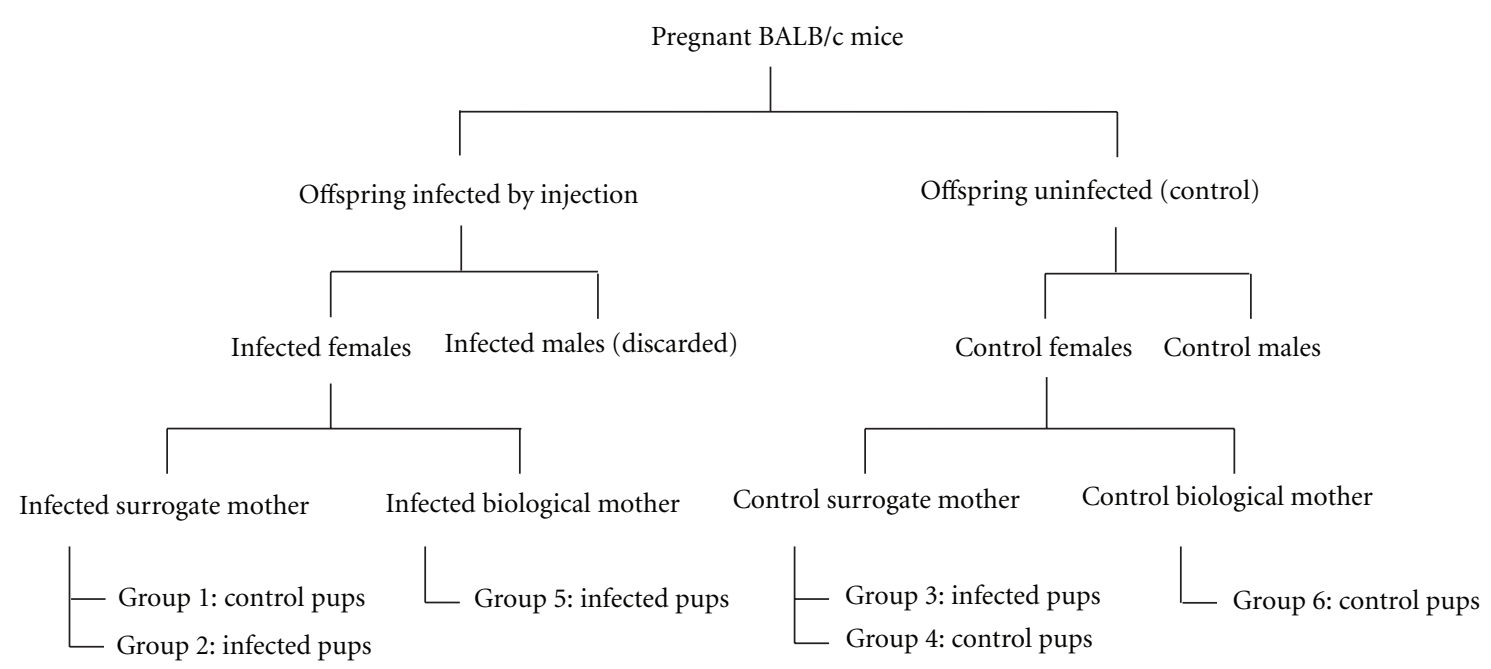

FIGURE 1: Experimental plan for ts-1 breast milk transmission studies.

rate in developing countries. Breastfeeding for 15 months accounts for $38 \%$ of the overall MTCT rate [27], and if continued for 24 months, it accounts for 44\% [28]. Richardson et al. [29] reported that if an infant ingests one liter of breast milk then the probability of having the HIV1 infection is similar to that of heterosexual transmission. Breast milk transmission depends on high maternal viral load and status of immunodeficiency with mastitis and duration of breastfeeding increasing the rate of HIV infection [29]. Breastfeeding by HIV positive women can reverse the benefits made in reducing perinatal HIV transmission through the use of HAART during pregnancy and/or labor. For example, in the Petra study of MTCT of HIV [30], the benefits of perinatal HAART prophylaxis were lost in the breastfeeding group. In order to prevent milk-borne HIV, HAART must be given throughout the breastfeeding period, which is rarely a feasible option in developing countries.

Studies in primates with HIV-2 [31], simian immunodeficiency virus (SIV) and SHIV (SIV expressing HIV envelope) $[32,33]$ have shown that primate lentiviruses can be transmitted orally. After milk is swallowed, gastric acid may inactivate some virus, but the buffering capacity of milk may allow the remaining virus to enter into the lumen of the gut, where there is extensive lymphoid tissue as a target for HIV. Mixed feeding (breastmilk and formula) carries a significantly increased risk of HIV transmission, because formula foods increase gut permeability [34]. These data suggest that the intestine could be a site of HIV entry in breast milk transmission, in addition to the oropharyngeal cavity. In areas without clean water, WHO recommends exclusively breastfeeding to avoid fatal diarrhea and the increased risk of HIV infection related to mixed feeding of formula and breast milk [35].

Several methods have been studied to decrease MTCT of HIV in areas where breastfeeding is a necessity. These include wet nursing by an HIV-negative woman, heat-treating the mother's milk with Holder pasteurization (62.5 for $30 \mathrm{~min})$, leaving milk at room temperature for 30 minutes to allow milk lipase to inactivate HIV, adding microbicides to milk and standing for 5-10 minutes, or getting milk from a human milk bank $[35,36]$. Due to high cost, obtrusiveness, inaccessibility, and/or inconvenience, none of these options are practical in developing countries where breastfeeding remains the only viable option for infant feeding and thus a major route of vertical HIV transmission. Therefore, a prophylaxis is urgently needed. However, in order to develop an effective prophylaxis, the exact mechanism of breast milk transmission of retrovirus must be clearly determined. Indepth study of the pathophysiology of retroviral transmission via breast milk using an animal model such as our ts1 model will provide mechanistic insights that can translate into effective human therapies.

2.3. Breast Milk Transmission of MoMuLVts-1 in BALB/c Mice. In the study of viral transmission by breast milk, use of 


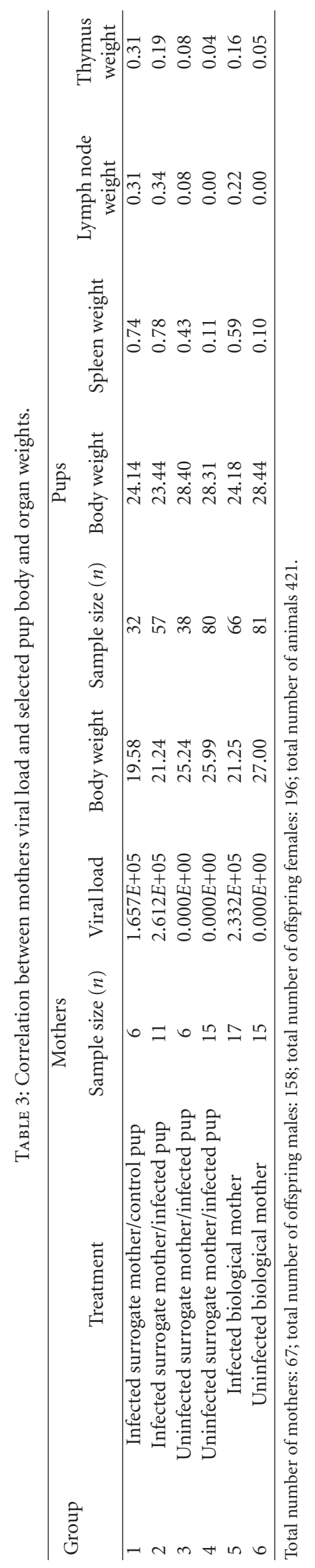




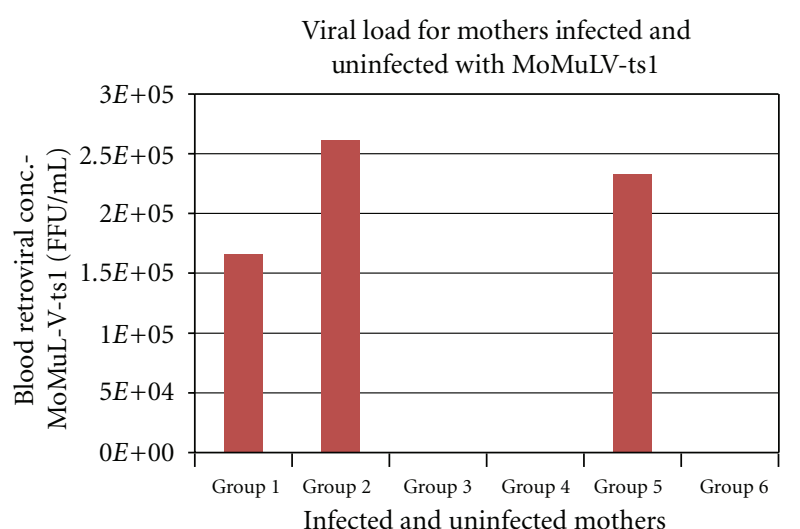

Viral load

(a)

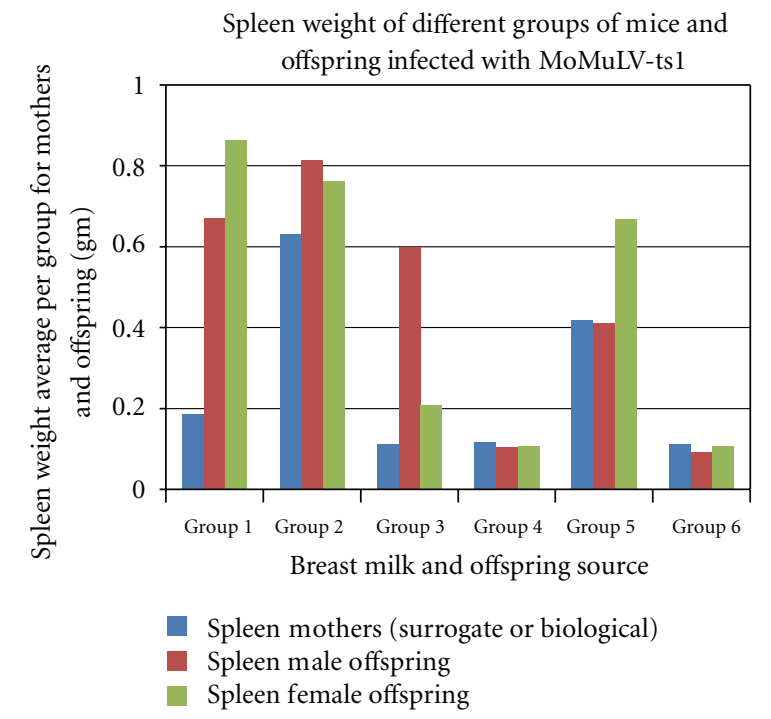

(c)

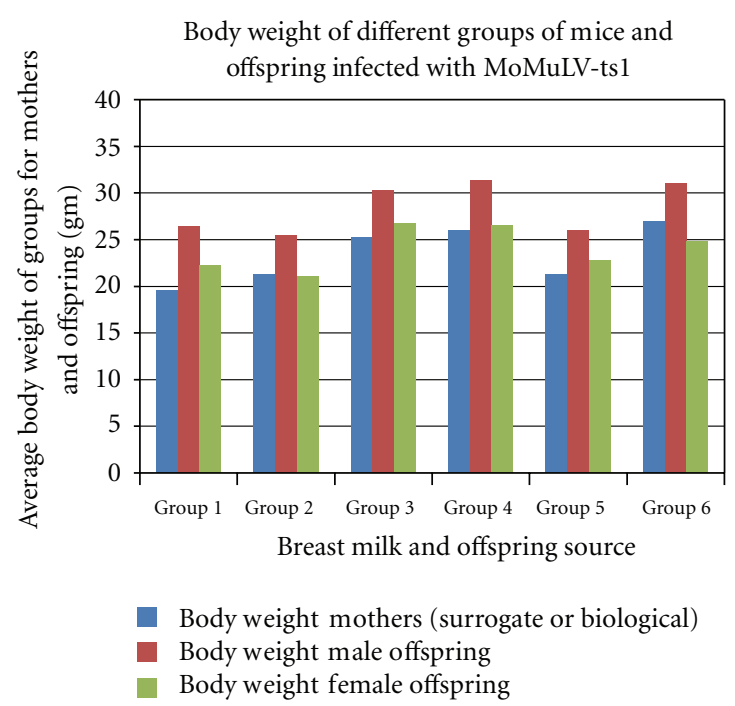

(b)

Mesenteric lymph node weight of different groups of mice and offspring infected with

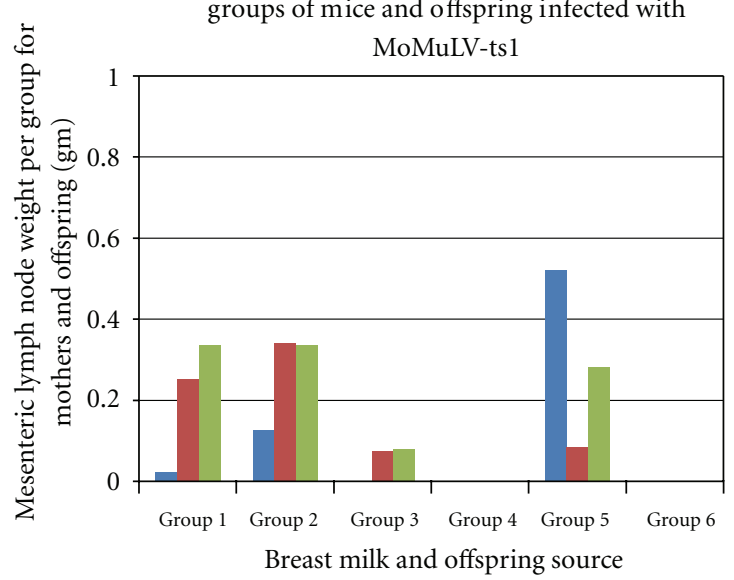

Lymph node mothers (surrogate or biological)

Lymph node male offspring

Lymph node female offspring

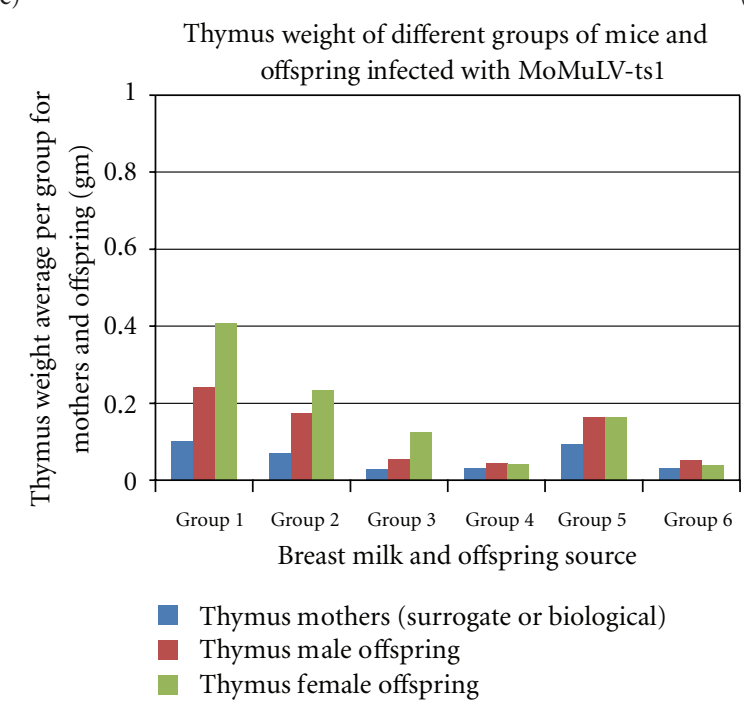

(d)

Thymus weight of different groups of mice and

) 


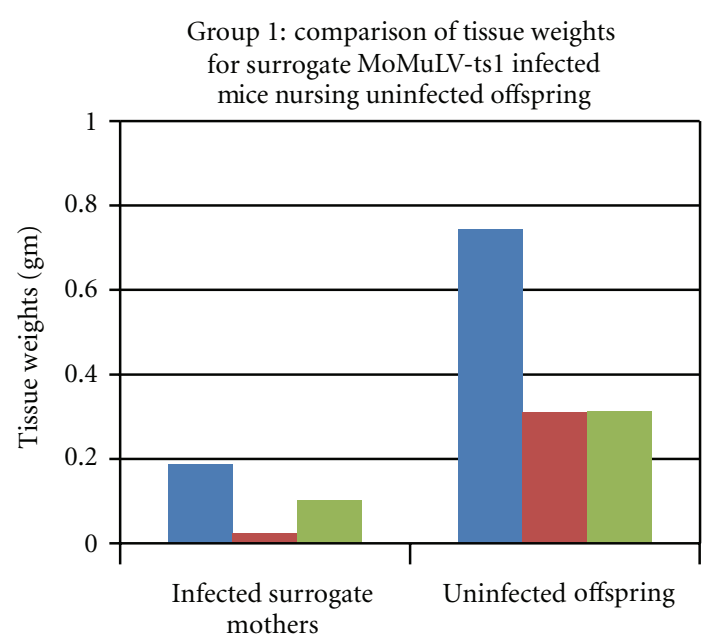

(a)

Group 3: comparison of tissue weights for uninfected surrogate mice nursing MoMuLV-ts1 infected offspring

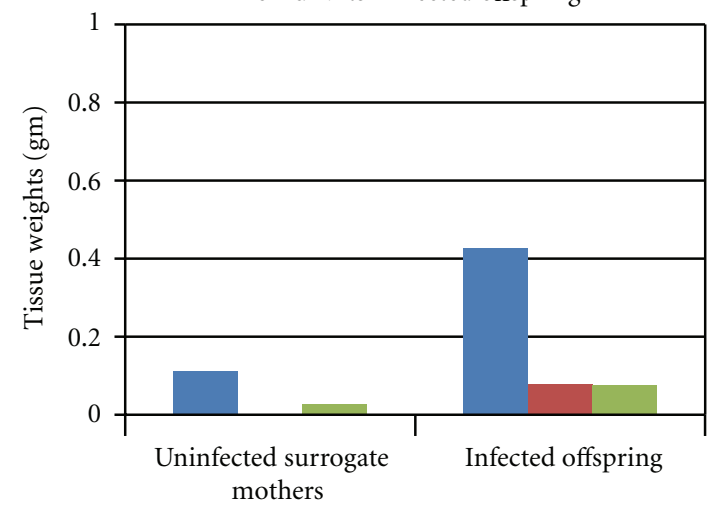

(c)

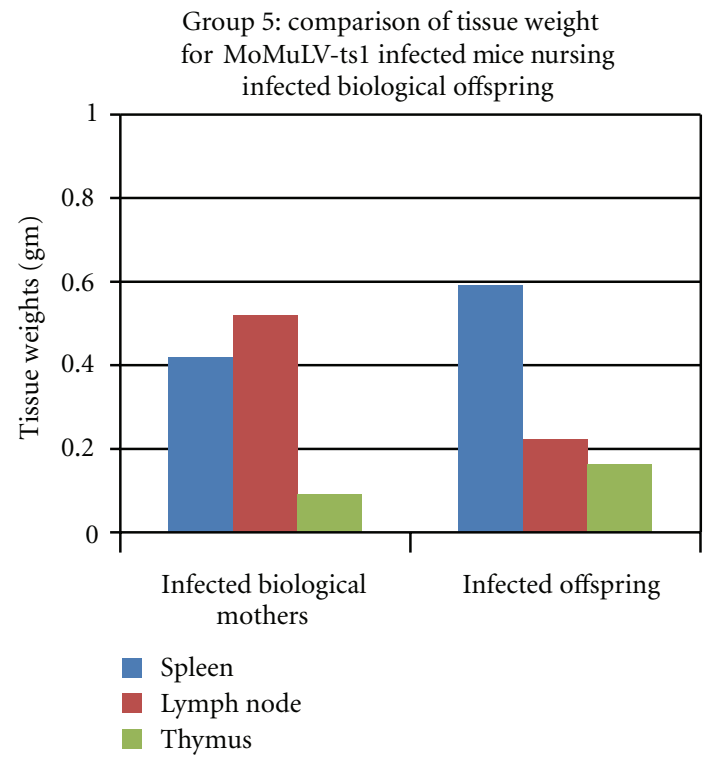

(e)

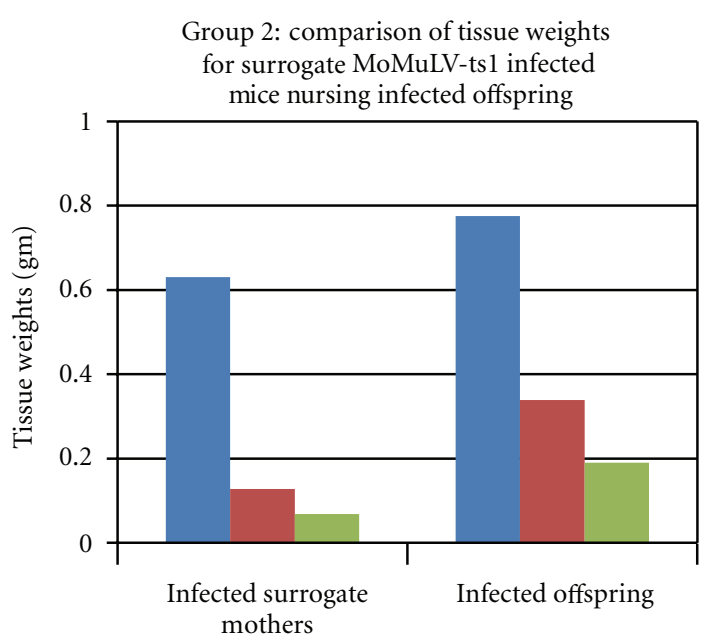

(b)

Group 4: comparison of tissue weight for uninfected surrogate mice nursing uninfected offspring

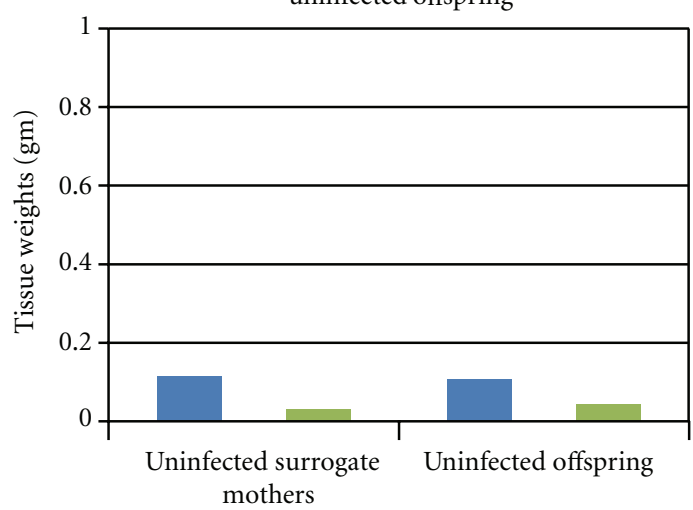

(d)

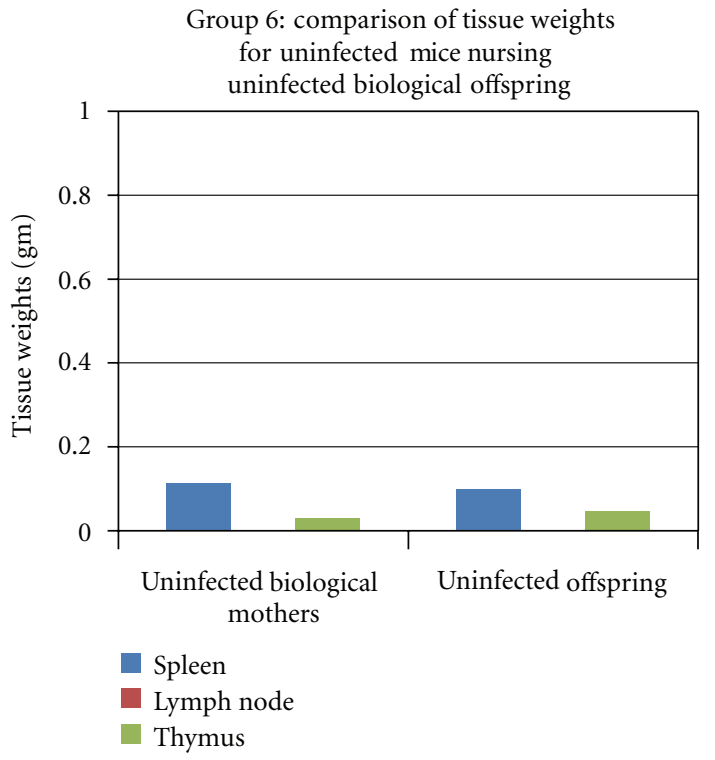

(f)

FIgURE 3: Tissue weight of mothers and offspring of each of 6 groups. 
TABle 4: Flow cytometric Analysis of CD4+, CD8+, and CD19 Cells.

\begin{tabular}{|c|c|c|c|c|}
\hline \multicolumn{5}{|c|}{ Flow Cytometric analysis } \\
\hline & & CD4 (T-cell) & CD8 (T-cell) & CD19 (B-cell) \\
\hline \multirow{3}{*}{ Blood } & Control & $15.4 \pm 10.6$ & $4.4 \pm 2.9$ & $2.6 \pm 1.2$ \\
\hline & Infected (no lymphoma) & $5.1 \pm 4.2$ & $1.0 \pm 0.7$ & $0.5 \pm 0.6$ \\
\hline & Infected (with lymphoma) & $3.2 \pm 2.4$ & $1.5 \pm 1.2$ & $1.7 \pm 1.3$ \\
\hline \multirow{3}{*}{ Spleen } & Control & $13.1 \pm 4.7$ & $4.9 \pm 2.0$ & $22.6 \pm 7.1$ \\
\hline & Infected (no lymphoma) & $12.5 \pm 0.8$ & $4.6 \pm 0.3$ & $10.1 \pm 2.4$ \\
\hline & Infected (with lymphoma) & $2.8 \pm 0.6$ & $1.8 \pm 1.2$ & $2.2 \pm 1.2$ \\
\hline \multirow{3}{*}{ Thymus } & Control & $15.6 \pm 5.6$ & $16.6 \pm 8.5$ & $0.9 \pm 0.2$ \\
\hline & Infected (no lymphoma) & $25.3 \pm 33.3$ & $1.2 \pm 0.1$ & $2.0 \pm 1.4$ \\
\hline & Infected (with lymphoma) & $8.6 \pm 9.2$ & $8.9 \pm 13.5$ & $0.4 \pm 0.5$ \\
\hline \multirow{3}{*}{ Lymph Node } & Control & $(\mathrm{NA})$ & (NA) & $(\mathrm{NA})$ \\
\hline & Infected (no lymphoma) & (NA) & (NA) & (NA) \\
\hline & Infected (with lymphoma) & $11.3 \pm 8.2$ & $4.3 \pm 3.6$ & $8.6 \pm 7.0$ \\
\hline
\end{tabular}

(NA): Not Available.

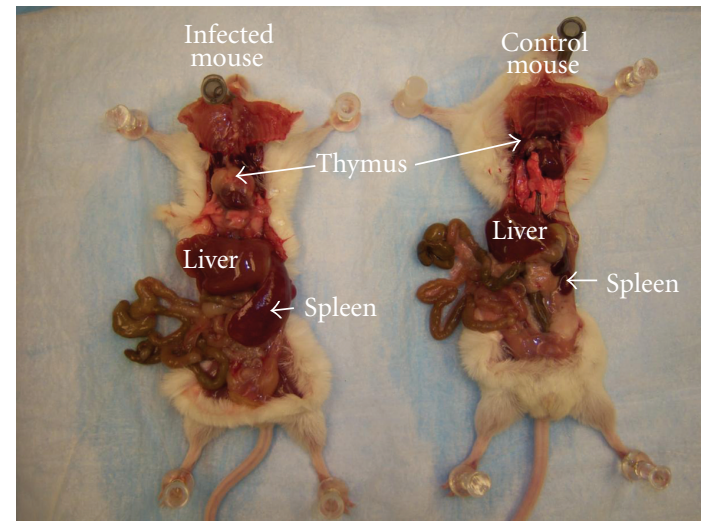

Figure 4: Organomegaly in infected versus control mice.

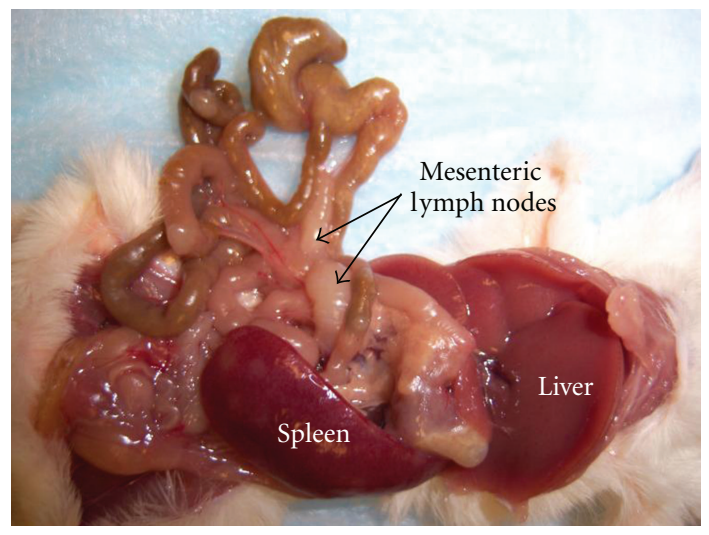

FIGURE 5: Manifestations of MoMuLV-ts-1 retroviral infection on different organ system.

a small animal model with a well-characterized immune system, such as the mouse, is ideal. As mentioned above, infection with ts- 1 results in an AIDS-like syndrome. When neonatal BALB/c mice less than 10 days old are injected intraperitoneally with ts-1, clinically apparent infection

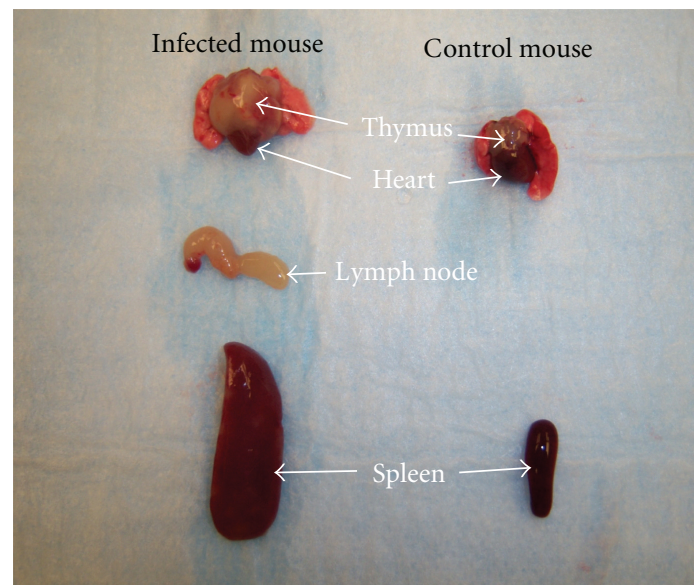

Figure 6: Excised organs from infected versus control mouse.

develops within 10 weeks. This clinical syndrome in mice is characterized by progressive bilateral hind limb paralysis, severe body wasting, and immunodeficiency [15]. Milder clinical illness can be induced by varying the dose of virus injected and/or the time of viral inoculation up to 10 days of age. CD4 cells are depleted by apoptosis, yielding AIDS-like clinical manifestations [12-14].

Several unique characteristics of this virus determine the experimental manner in which it is evaluated. The infectivity of this virus is strain specific and dependent upon the age of the mouse at the time of exposure, but the temperature sensitive nature of the virus does not affect its in vivo infectivity $[15,37]$. BALB/c mice are a susceptible strain, with clinical diseases developing in $98 \%$ of mice by week 10 after intraperitoneal injection. Viral injection of mice after ten days of age does not result in clinically detectable disease but does result in antibody production $[19,38]$. Perinatal transmission of ts-1 occurs in utero, intrapartum, and via breastfeeding $[2,3]$. Breastfeeding is a highly effective route of MTCT in this model: $95 \%$ to $99 \%$ of uninfected neonatal 


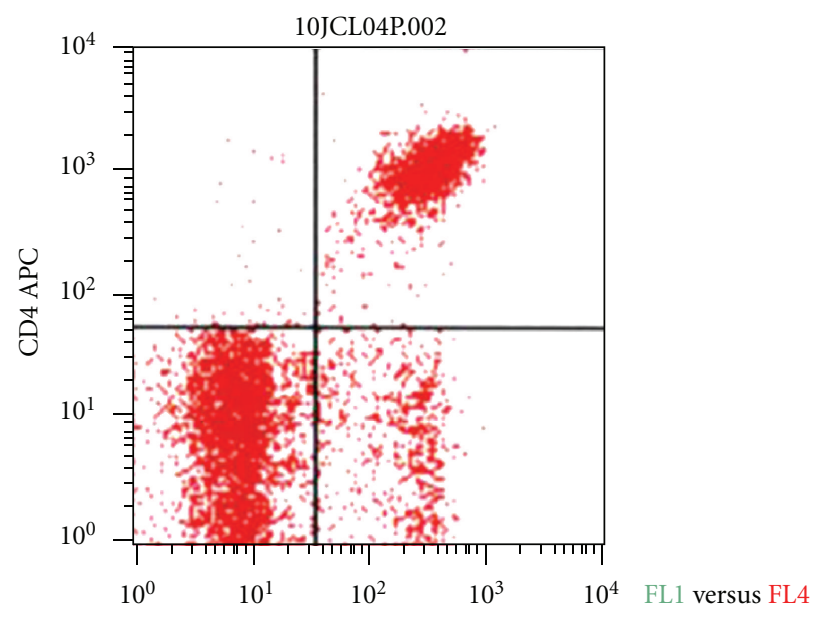

CD3 FITC

\begin{tabular}{crrrrr} 
Quad & Events & Gated (\%) & Total (\%) & $X$ mean & $Y$ mean \\
\hline UL & 39 & 0.66 & 0.24 & 12.3 & 337.3 \\
UR & 1406 & 23.69 & 8.8 & 362.75 & 1156.95 \\
LL & 3669 & 61.83 & 22.97 & 8.93 & 10.49 \\
LR & 820 & 13.82 & 5.13 & 226.42 & 9.21
\end{tabular}

$\mathrm{CD}_{4}$ cells from control offspring

(a)

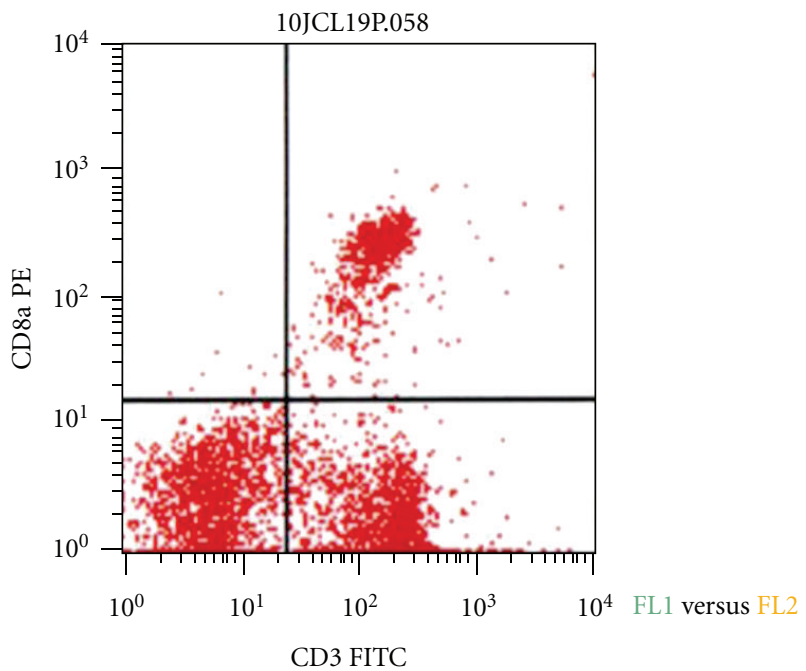

CD3 FITC

\begin{tabular}{crrrrr} 
Quad & Events & Gated (\%) & Total (\%) & $X$ mean & $Y$ mean \\
\hline UL & 11 & 0.2 & 0.11 & 11.21 & 31.72 \\
UR & 781 & 14.39 & 7.66 & 187.62 & 232.14 \\
LL & 1615 & 29.75 & 15.83 & 7.02 & 3.27 \\
LR & 3022 & 55.66 & 29.63 & 220.23 & 1.67
\end{tabular}

$\mathrm{CD}_{8}$ cells from control offspring

(c)

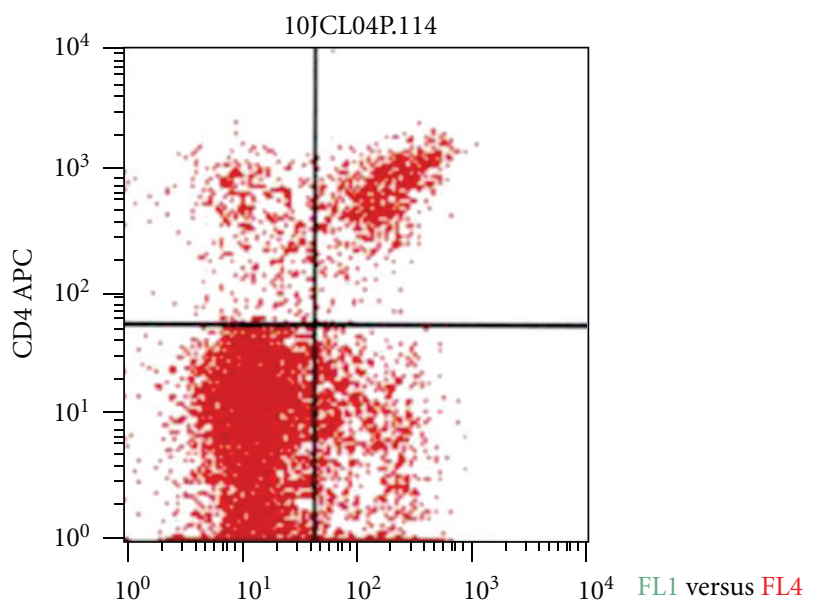

CD3 FITC

Quad Events Gated (\%) Total (\%) $X$ mean $Y$ mean

\begin{tabular}{rrrrrr}
\hline UL & 415 & 5.42 & 1.26 & 16.8 & 488.74 \\
UR & 697 & 9.11 & 2.11 & 206.42 & 740.79 \\
LL & 5473 & 71.54 & 16.55 & 14.73 & 10.44 \\
LR & 1065 & 13.92 & 3.22 & 144.47 & 9.11
\end{tabular}

$\mathrm{CD}_{4}$ cells from infected offspring

(b)

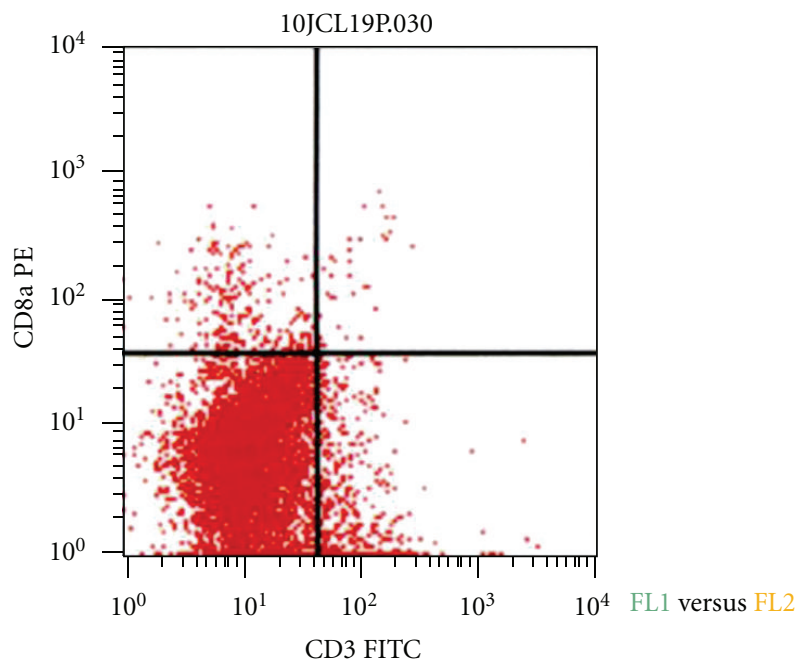

\begin{tabular}{crrrrr} 
Quad & Events & Gated (\%) & Total (\%) & $X$ mean & $Y$ mean \\
\hline UL & 231 & 3.12 & 2.06 & 14.35 & 108.22 \\
UR & 42 & 0.57 & 0.37 & 94.03 & 201.36 \\
LL & 6511 & 88.06 & 58.03 & 13.73 & 8.04 \\
LR & 610 & 8.25 & 5.44 & 125.47 & 5.18
\end{tabular}

$\mathrm{CD}_{8}$ cells from infected offspring

(d)

FIgURE 7: Examples of the flow cytometric analysis showing frequency of CD4+ and CD8+ cells from control and infected offspring. Samples were tagged with CD3 FITC, CD4 APC and CD3 FITC, CD8 PE. Upper left quadrant (UL) shows naïve lymphocytes. Upper right quadrant (UR) shows CD3+/CD4+ or CD3+/CD8+ frequency for cells positive for both CD3 and CD4 or CD3 and CD8 markers. Lower left quadrant (LL) shows CD3-/CD4- or CD3-/CD8 - frequency for cells negative for both CD3/CD4 or CD3/CD8 markers. Lower right quadrant (LR) shows cells positive for $\mathrm{CD} 3$ markers only. 
TABLE 5: Retrovirus genera and type of species affected.

\begin{tabular}{|c|c|c|}
\hline \multicolumn{3}{|c|}{ Classification of retroviruses } \\
\hline Genus & Species & Examples \\
\hline Alpharetrovirus & Birds & Avian leukosis viruses (AVL), Rous sarcoma virus (RSV) \\
\hline Betaretrovirus & Mice, primates, and sheep & $\begin{array}{l}\text { Mouse mammary tumor virus (MMTV), Mason-Pfizer monkey virus } \\
\text { (MPMV), and Jaagsiekte sheep retrovirus (JSRV) }\end{array}$ \\
\hline Gammaretrovirus & Mice, cats, primates, and birds & $\begin{array}{l}\text { Murine leukemia virus (MLV), feline leukemia viruses, gibbon ape leukemia } \\
\text { virus, reticuloendotheliosis virus, and xenotropic murine retrovirus (xMRV) }\end{array}$ \\
\hline Deltaretrovirus & Cattle and primates & Bovine leukemia virus, human T-lymphotropic virus \\
\hline Epsilonretrovirus & Fish & Walleye dermal sarcoma virus \\
\hline Lentivirus & Primates, sheep, cats, and horses & $\begin{array}{l}\text { Human immunodeficiency virus (HIV), simian immunodeficiency virus } \\
\text { (SIV), Maedi/visna virus, feline immunodeficiency virus (FIV), and equine } \\
\text { infectious anemia virus }\end{array}$ \\
\hline Spumavirus & Primates, cats, and cattle & $\begin{array}{l}\text { Human foamy virus (HFV), simian foamy virus (SFV), feline foamy virus, } \\
\text { and bovine foamy virus }\end{array}$ \\
\hline
\end{tabular}
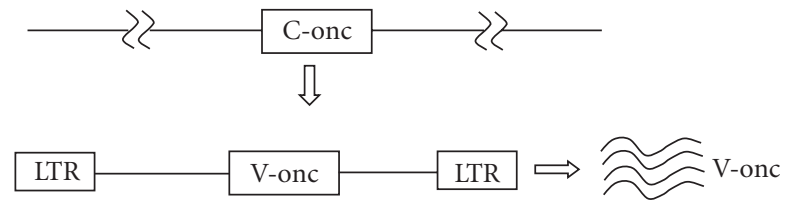

FIGURE 8: Acute transforming virus, capture of a c-onc and overexpression of $\mathrm{v}$-onc by provirus.

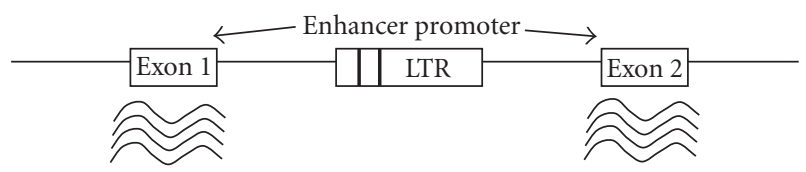

FIgURE 9: Enhancer of promoter insertion either upstream or downstream of growth controlling cellular genes.

mice, if suckled from an infected mother, develop clinically symptomatic ts- 1 infection $[3,4]$.

Our laboratory was the first to clearly demonstrate that breast milk transmission in offspring of ts- 1 infected BALB/c mice causing AIDS like condition. We have established a mouse model for MTCT for MoMuLV ts-1 for in utero, intrapartum, and postpartum (breastfeeding) routes of transmission $[2-4,23]$. The following information provide some murine model data for retroviral transmission via breastfeeding. Pregnant BALB/c female mice delivered pups which were divided into experimental and control groups. Seventy two hours after birth, the pups were injected intraperitoneally (ip) with $0.1 \mathrm{~mL}$ of $4.0 \times 10^{6} \mathrm{ffu} / \mathrm{mL}$ ts- 1 virus and control pups were injected with $0.1 \mathrm{~mL}$ DMEM medium [2-4]. These mice were allowed to mate with uninfected males and produce offspring. Within $10-12 \mathrm{~h}$ after birth, offspring suckled either from control or ts1infected surrogate or biological mothers (Figure 1). Tissue collection, histology, electron microscopy, flow cytometry, DNA extraction, PCR, and sequencing of viral DNA were performed. Four hundred twenty one mice were divided into 6 groups as described in our previous paper [3]. Pups from the ts- 1 control mothers suckled from the infected surrogate mothers (Group 1), pups from the infected mothers suckled from the infected surrogate mothers (Group 2), or from infected biological mothers (Group 5). In Group 3, infected pups suckled from the control mothers. Groups 4 and 6 were controls. Group 4 control pups suckled from the control surrogate mothers, and Group 6, control pups suckled from their control biological mothers.

The rate of postpartum ts- 1 transmission in this study was almost $100 \%$. This rate is much higher than the MTCT of HIV in infants via breastfeeding (about 20\%-30\%), which is advantageous in an experimental animal model. Maternal viral load appear to correlate with development of lymphoma (Table 3; Figures 2 and 3), but further studies are needed to verify this. The figures and tables listed below show some of the gross morphology and flow cytometric data. Significant and consistent increases in weights and change in gross morphology of spleen, lymph node, and thymus (Tables 2 and 3; Figures 4, 5, and 6) are readily apparent. Our flow cytometric data shows decreases in CD4 and CD8 (Figure 7) cell population in the advanced stages of lymphoma development. In addition, decreasing numbers of CD8 and B-cells have been shown (Table 4) in the presence of clinically apparent immunodeficiency and wasting.

\section{Part II. ts-1 and Lymphomagenesis}

3.1. Retrovirus Induced Cancer. The first reports of retroviruses associated with cancer occurred in the early 20th century when an avian erythroblastosis virus (AEV) was isolated from spontaneous erythroleukemia in a chicken [39]. Shortly after that, Peyton Rous demonstrated that chicken sarcomas were infectious and could induce tumors when transmitted into healthy birds [40]. These novel observations were followed by multiple reports of retroviruses isolated from a broad range of mammals such as rodents, cats, sheep, and cows in association with both malignancies and immunodeficiencies. The first human retrovirus, human Tlymphotropic virus type 1 (HTLV-1), was isolated in 1980 [41] and has been shown to induce adult T-cell leukemia (ATL) [42]. 


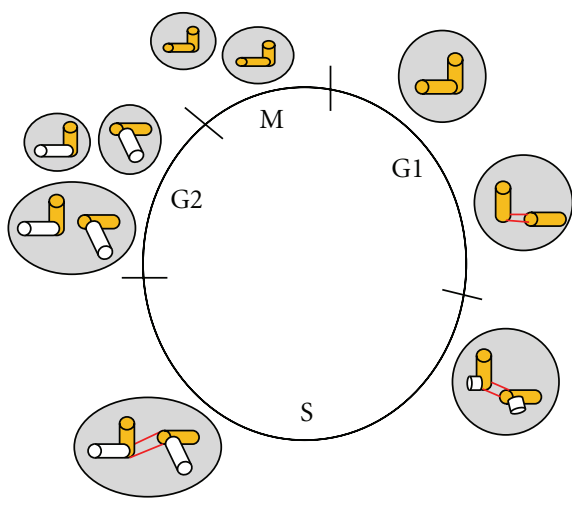

(a) Stages of cell cycle

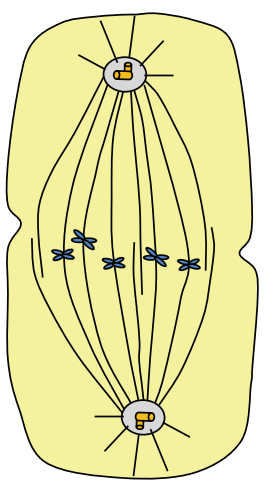

(b) Animal cell (centriole present)

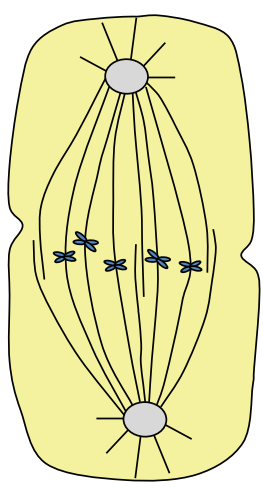

(c) First cleavage division of a mammalian egg (no centriole)

FIGURE 10: This figure shows the centriole-centrosome division during cell cycle (a), presence of both centriole and centrosome in the animal cell (b), and absence of centriole in the first cleavage division of a mammalian egg (c).

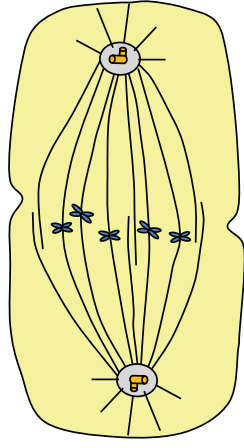

Normal division

(a)

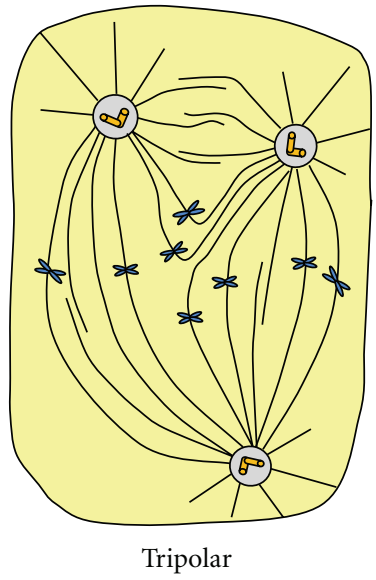

(b)

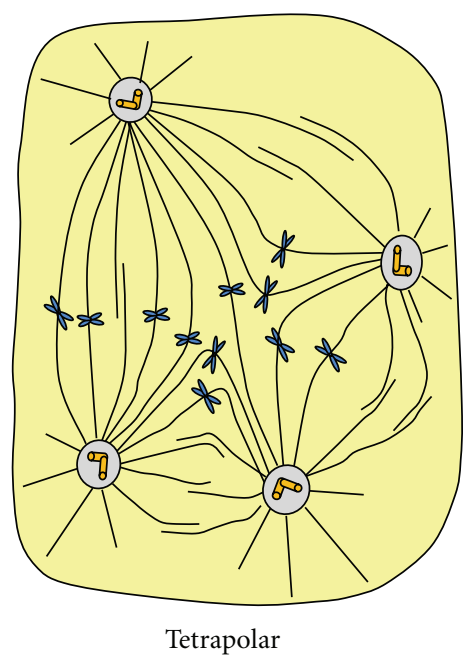

(c)

FIGURE 11: Normal and abnormal cell division due to abnormal centriole-centrosome division. As a result, giant cells with multiple nuclei may be present in a malignant cell.

Retroviruses are classified into seven genera. Oncogenic retroviruses (retroviruses that induce tumorigenesis) belong to the following genera (Table 5) $[43,44]$.

Oncogenic retroviruses which induce tumors can be divided into two classes: acute and slow transforming viruses. Acute transforming retroviruses induce polyclonal tumors within 2 to 3 weeks after infection of the host. These retroviruses induce tumors through acquisition and overexpression of cellular proto-oncogenes that have captured virus (Figure 8) [45]. An example is $\mathrm{v}-\mathrm{Abl}$ in the Abelson Murine Leukemia Virus [46].

In contrast, slow transforming retroviruses induce mono- or oligoclonal tumors with a longer latency of several months. These types of retroviruses do not carry viral oncogenes and can cause tumors by activating cellular protooncogenes close to the proviral DNA integration site on the host genome (Figure 9).
Elements in the proviral genome that regulate the viral transcript also act at common integration sites (cis) on cellular gene transcripts. Depending on whether the provirus integrates into the genes or in the vicinity of the genes, these elements can enhance or disrupt normal transcription, and thus induce oncogenic mutations. These classes of retroviruses have been found to induce tumors in many animals, including birds (ALV and REV), and mice (MLV); [45]. In addition to these two general transformation groups, a small number of retroviruses induce tumors by expression of their own oncogenic proteins. For example, human Tcell leukemia virus types 1 and 2 (HTLV-1 and HTLV2 , resp.) induce adult T-cell immortalization and leukemia in human by expression of viral Tax protein. Tax has no cellular homologue, and it works in trans to disrupt cellular checkpoints and destabilize genome integrity [47] leading to transformations that directly cause human cancer [48]. 
TABLE 6: Gene expression analysis of the spleen tissues were used to investigate the centriole-centrosome pathway for infected versus control mice. tacc3 showed the highest upregulation with 30.13-fold increase, much more than our previous report [4]. tp $x 2$ and $p 53$ showed significant downregulation. During this investigation, aurka did not show significant upregulation. This is due to the variation in this group of six mice used in this study. However, a trend of upregulation of aurka is evident in 3 mice in column 2.

\begin{tabular}{|c|c|c|c|c|c|c|}
\hline \multicolumn{7}{|c|}{ Gene expression } \\
\hline Mouse ID & tacc3 & aurka & ndel1 & $t p \times 2$ & p53 & rhamm \\
\hline Control 1 & (NA) & (NA) & 1.18 & (NA) & (NA) & (NA) \\
\hline Control 2 & (NA) & (NA) & 0.17 & (NA) & 0.45 & 0.56 \\
\hline Control 3 & 2.32 & 0.8 & 0.42 & 0.91 & 0.66 & 0.75 \\
\hline Control 4 & 0.37 & 0.96 & 2.78 & 1.85 & 1.95 & 1.53 \\
\hline Control 5 & 0.85 & 0.96 & 0.57 & 1.19 & 0.93 & 1.17 \\
\hline Control 6 & 0.64 & 1.52 & 0.09 & 0.17 & (NA) & (NA) \\
\hline Control 7 & 0.82 & 0.76 & 1.81 & 0.88 & (NA) & (NA) \\
\hline SEM & 0.34 & 0.14 & 0.37 & 0.27 & 0.33 & 0.22 \\
\hline $\begin{array}{l}\text { Normalized avg. } \\
\text { fold Exp. }\end{array}$ & 1.00 & 1.00 & 1.00 & 1.00 & 1.00 & 1.00 \\
\hline Lymphoma 1 & 11.25 & 1.25 & 0.14 & 0.15 & 0.26 & 1.63 \\
\hline Lymphoma 2 & (NA) & 0.44 & 0.12 & 0.2 & 0.13 & 0.67 \\
\hline Lymphoma 3 & (NA) & 0.4 & 0.25 & 0.11 & 0.15 & 1.63 \\
\hline Lymphoma 4 & 53.53 & 2.41 & 0.2 & 0.15 & 0.71 & 0.88 \\
\hline Lymphoma 5 & 53.16 & 1.87 & 0.24 & 0.15 & 0.6 & 0.9 \\
\hline Lymphoma 6 & 2.57 & (NA) & 0.09 & 0.15 & 0.07 & 0.85 \\
\hline Avg Fold Exp. & 30.13 & 1.27 & 0.17 & 0.15 & 0.32 & 1.09 \\
\hline SEM & 13.52 & 0.39 & 0.03 & 0.01 & 0.11 & 0.17 \\
\hline$P \leq 0.05$ & 0.04 & 0.53 & 0.07 & 0.01 & 0.05 & 0.75 \\
\hline
\end{tabular}

(NA): data not available.

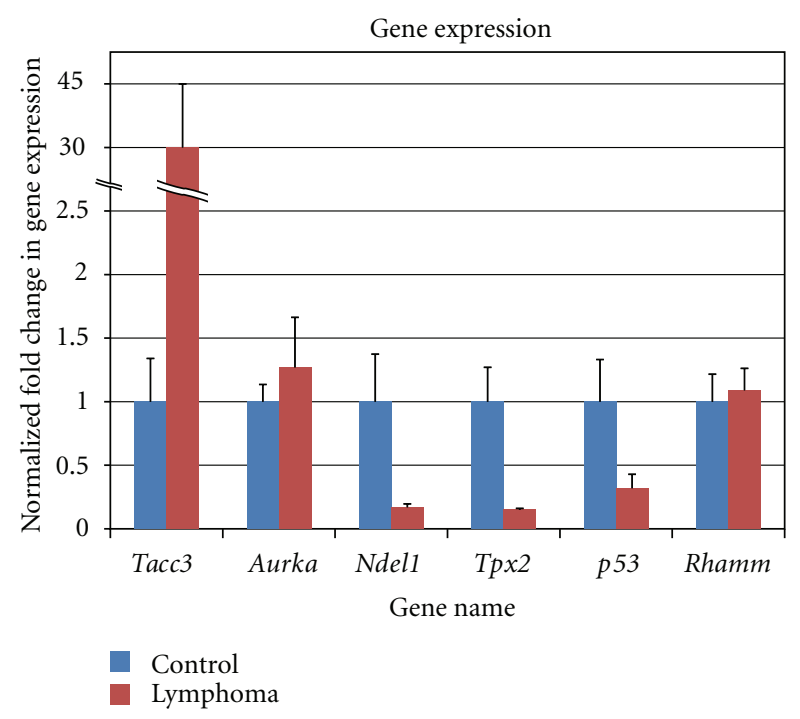

FIGURE 12: Graph of fold change in gene expression versus normalized control.

In AIDS-related lymphoma, patient studies indicate that oncogene activation by insertional mutagenesis of the HIV might be another mechanism by which HIV-1 can induce cancer $[49,50]$.

The release of the complete mouse genome sequence and the availability of reliable methods for isolation of proviral flanks have introduced the retroviral insertion mutagenesis screen in mice as a powerful procedure to identify genes contributing to tumorigenesis. Many oncogenes identified in these screens have given a valuable basis for better understanding the development of human cancer [45]. The MLV utilizes a slow transformation mechanism to induce leukemia or lymphoma in mice and is one of the retroviruses that provides an excellent model to identify and study the oncogenes involved in retrovirus-induced tumorigenesis.

3.2. Centrosomal Involvement in Cell Cycle and Cancer. Centriole-centrosome is the microtubular organization center (MTOC) and plays a major role during mitosis for chromosomal arrangement at the equatorial plane during metaphase and pulling the chromatids in two opposite spindle poles during anaphase. Spindles start to organize as soon as centrioles divide and two centrosomes are formed. The spindle microtubules act like railroad tracks on which the chromatids travel towards the poles with the help of several motor proteins including dynein and kinesin. However, the centriole is not an essential structure for many cells including first cleavage division of mammalian eggs [51]. Figure 10 describes the centrosome-centriole cycle in a typical animal cell at mitosis and the first cleavage division of a mammalian egg without the centriole. The centrosomewhich is an accumulation of amorphous materials around the centriole-is present in most mammalian cells. In these 


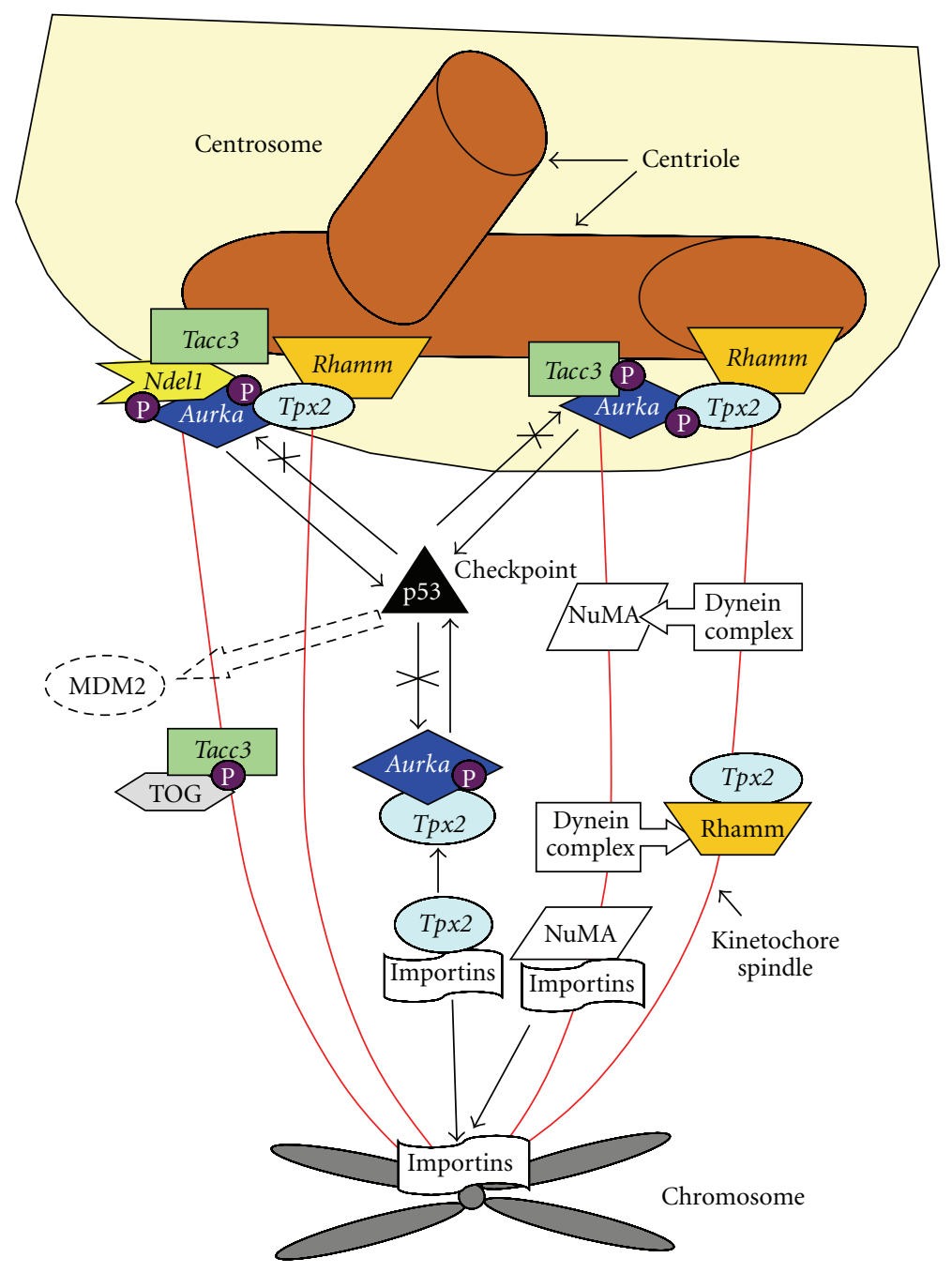

FIGURE 13: Pathway of Genes associated with spindle formation.

cells, the centrosome is an essential component in the development of spindle poles and progression to mitosis and cytokinesis. There are three types of spindles: kinetochore, interpolar, and astral. The kinetochore spindle extends from poles to chromosomes. The interpolar spindles run from pole to pole with no connection to the chromosomes, while the astral spindles run between centrosomes to the cell cortex. Thus, three types of spindles play different roles.

There are numerous centrosomal proteins and new proteins are being discovered every year. Although the centriolecentrosome system is essential for cell cycle and normal chromosomal segregation, little is known about the intricate details of this structure, which is extremely important in the understanding of cancer development and progression. For example, many cancers show abnormal mitosis leading to tetraploidy and aneuploidy. According to Wang et al. [52], many carcinomas may have either or both structural and numerical abnormalities; this occurs during early stage of tumorigenesis. This is also correlated with chromosomal instability and tumor progression. Numerical chromosomal abnormalities can also occur due to bipolar and tripolar or tetrapolar spindle formation (Figure 11).
Unequal segregation of chromosomes leads to multiploidy and/or aneuploidy. Viral gene insertion into the normal genome close to the centrosomal genes may lead to abnormal mRNA expression resulting in abnormal protein production and activity. For example, increases in Tacc3 and Aurora A kinase (Aurka) can produce tumor like cells $[53,54]$.

3.3. Insertion of Viral Genome into the Mouse Genome Causing Abnormal Centrosomal Protein Production Leading to Lymphomagenesis. A large number of investigations have been carried out to study the development of lymphoma by MoMuLV, but so far, little data are available on the natural transmission of this retrovirus by breast milk causing lymphoma. Extensive studies are needed on breast milk transmitted retroviruses, since evidence exists that viruses such as HTLV-1 or Epstein-Barr virus (EBV) may be oncogenic and also have epidemiologic patterns indicating perinatal or early childhood transmission. The best studied of these viruses is EBV. Studies have shown that EBV and MLV gene insertion into the host genome alters gene 
expression leading to carcinogenesis [55-59]. We have used inverse PCR (I-PCR), DNA cloning, sequencing, and quantitative reverse transcriptase-PCR (qRT-PCR) to study the viral integration into mouse genome. Tissue samples from spleen and lymph nodes were used for the genomic studies [4]. Using the primary PCR product as template, secondary PCR was performed. After the PCR bands were obtained, cloning was done and colonies with viral inserts were located and analyzed. The differential expression of candidate genes was obtained by using quantitative realtime PCR. Gene specific primers were designed using Primer Express software. GAPDH was used as "housekeeping" gene to confirm amplification factor for each PCR product meltcurve analysis process. RNA expression levels were calculated by using ddCt method. Gene expression were recorded by mRNA expression and reported as "fold change". Statistical analyses were performed by using statistical package of the social sciences (SPSS). 35 genes were selected for mRNA expression based on preselected criteria. Table 6 and Figure 12 show the change in mRNA expression of six genes Tacc3 shows the highest upregulation with 30.13-fold increase, much more than our previous report [4].

How are changes in gene expression associated with lymphoma in this murine model? In our mouse model, about $50 \%$ of pups develop lymphoma. This high incidence of lymphoma may be due to the genomic integration of viral DNA into the mouse genome causing changes in nearby genes. While viral gene insertion appears to be random, in pups that develop lymphoma, there is a predilection for viral insertion near genes involved in spindle formation. One of the mechanisms of lymphoma development, therefore, may be the alteration in spindle assembly-disassembly pathway. Up or down regulation of these genes may occur, breaking the balance of protein production, and causing abnormality in spindle formation, chromosomal segregation, and subsequent lymphoma production. Although there are many genes involved in the spindle assembly pathway, we have studied 6 genes predominately associated with viral genomic integration in mice with lymphoma including tacc3, aurka, tpx2, rhamm, ndel1 and p53. Of these 6 genes, we have observed the highest fold increase of tacc 3 followed by aurka. Figure 13 shows the proteins (gene product) associated with spindle formation.

All tumor samples showed increases in mRNA expression of tacc3 gene compared to the control group. Out of the 6 genes reported in this study, two were upregulated, including tacc3 and aurka. As previously reported, tacc3 showed the highest mRNA expression levels with an average of 9.16-fold increase [4]. tacc3 is associated with centrosome and microtubule-associated proteins that are essential for mitotic spindle formation [60] and have been associated with dysfunction in a variety of tumors. Tacc3 was also identified as a novel prognostic marker in nonsmall cell lung cancer [61]. Dysregulation of Tacc3 proteins have also been related to ovarian cancer [62]. Schnieder et al. [63] demonstrated the important role of Tacc3 in spindle assembly and cellular survival, thus introducing it as a potential therapeutic target in cancer cells. Deficiency of Tacc3 leads to p53-mediated apoptosis [64]. Therefore, overexpression of tacc 3 , as observed in our mouse model may cause the downregulation of $p 53$, causing inhibition of apoptosis, thus leading to lymphoma.

Aurka connects microtubules to kinetochore and phosphorylates Tacc3, leading to its localization to spindle microtubules during metaphase and promoting its growth from centrosomes. Aurka is essential for accurate chromosome segregation. Overexpression of Aurka leads to spindle defects, aneuploidy, and tumor formation. Tpx2 and Rhamm helps with stable bipolar spindle formation through its association with Aurka [65-67]. Decrease in expression of these proteins may cause spindle fragmentation, while increased expression may cause disorganized, multipolar spindles coupled with inability to appropriately align and segregate chromatids. Ndel1 has a high affinity for Tacc3, and its disruption decreases centrosome targeting of Tacc3 [68]. p53 is a cellcycle checkpoint protein. DNA damage generally results in phosphorylation of $p 53$ leading to its dissociation from MDM2 and acts as a transcription factor, arresting the cells in the G1 phase of the cell cycle. Mutated or downregulated p53 results in continued proliferation in damaged cells and subsequent development of cancer. Upregulation of aurka and tacc 3 and downregulation of p53 are consistent with abnormal cell division, aneuploidy, and tumorigenesis. Downregulation of $t p \times 2$ is associated with abnormal spindle formation with the presence of giant cells and the absence of mitotic figures in histological specimens of lymphoma tissue. However, spindle pathway disruption is not the only mechanism by which malignancies develop or progress, but it may be an important factor for breast milk-transmitted retrovirus-induced cancer in the ts- 1 mouse model.

\section{Conclusion}

A well-developed animal model can be extremely important in the study of viral-induced cancer and immunodeficiency. Although there are obvious differences between murine and human cells, an appropriate mouse model can provide important clues to the possible pathways of molecular mechanisms of retroviral transmission and cancer development and progression. While this paper reviews ts- 1 as a small animal model of perinatal HIV transmission and ts- 1 in lymphomagenesis, it also raises a number of questions that need further study. We believe that this is a unique murine model for the study of retrovirusinduced lymphomagenesis for multiple reasons. Mice have been used extensively for genetic, physiological, biochemical, immunological, endocrinological, and reproductive research and an enormous body of background data-including the entire murine genome-is already available. In our unique murine model, breast milk transmission is near $100 \%$, but lymphoma development occurs in only $50 \%$ of infected pups across all litters; therefore, an ideal internal control exists in which to study causative genetic factors. Study of this model may contribute to the understanding of molecular mechanisms of spindle formation, chromosomal segregation, and interactions between many centrosomal proteins and may help clarify some of the cellular abnormalities leading to 
cancer. Some of these proteins and their inhibitors can be used for diagnostic and/or therapeutic development.

\section{Acknowledgment}

This work was supported by F. M. Douglas Foundation of St. Vincent Medical Center.

\section{References}

[1] UNAIDS and WHO, 2008, AIDS epidemic update, Joint United Nations Program on HIV/AIDS.

[2] J. Duggan, H. Okonta, and J. Chakraborty, "Vertical transmission of a murine retrovirus, ts1," Viral Immunology, vol. 17, no. 2, pp. 266-278, 2004.

[3] J. Duggan, H. Okonta, and J. Chakraborty, "Transmission of Moloney murine leukemia virus (ts-1) by breast milk," Journal of General Virology, vol. 87, no. 9, pp. 2679-2684, 2006.

[4] J. Chakraborty, B. Hussein, H. Bagalb et al., "Retroviral gene insertion in breast milk mediated lymphomagenesis," Virology, vol. 377, no. 1, pp. 100-109, 2008.

[5] P. K. Y. Wong, L. J. Russ, and J. A. McCarter, "Rapid, selective procedure for isolation of spontaneous temperature-sensitive mutants of Moloney leukemia virus," Virology, vol. 51, no. 2, pp. 424-431, 1973.

[6] P. K. Y. Wong and J. A. McCarter, "Studies of two temperature sensitive mutants of Moloney murine leukemia virus," Virology, vol. 58, no. 2, pp. 396-408, 1974.

[7] P. K. Y. Wong, M. M. Soong, R. MacLeod, G. E. Gallick, and P. H. Yuen, "A group of temperature-sensitive mutants of Moloney leukemia virus which is defective in cleavage of env precursor polypeptide in infected cells also induces hind-limb paralysis in newborn CFW/D mice," Virology, vol. 125, no. 2, pp. 513-518, 1983.

[8] P. K. Y. Wong and J. A. McCarter, "Genetic studies of temperature sensitive mutants of Moloney murine leukemia virus," Virology, vol. 53, no. 2, pp. 319-326, 1973.

[9] P. K. Y. Wong, C. Knupp, P. H. Yuen, M. M. Soong, J. F. Zachary, and W. A. F. Tompkins, "ts-1, a paralytogenic mutant of Moloney murine leukemia virus TB, has an enhanced ability to replicate in the central nervous system and primary nerve cell culture," Journal of Virology, vol. 55, no. 3, pp. 760-767, 1985.

[10] S. Clark, J. Duggan, and J. Chakraborty, "ts1 and LP-BM5: a comparison of two murine retrovirus models for HIV," Viral Immunology, vol. 14, no. 2, pp. 95-109, 2001.

[11] P. H. Yuen and P. F. Szurek, "The reduced virulence of the thymotropic Moloney murine leukemia virus derivative MoMuLV-TB is mapped to 11 mutations within the $\mathrm{U} 3$ region of the long terminal repeat," Journal of Virology, vol. 63, no. 2, pp. 471-480, 1989.

[12] K. Saha and P. K. Y. Wong, "ts1, a temperature-sensitive mutant of Moloney murine leukemia virus TB, can infect both $\mathrm{CD}^{+}$and $\mathrm{CD}^{+} \mathrm{T}$ cells but requires $\mathrm{CD} 4^{+} \mathrm{T}$ cells in order to cause paralysis and immunodeficiency," Journal of Virology, vol. 66, pp. 2639-2646, 1992.

[13] K. Saha, P. H. Yuen, and P. K. Y. Wong, "Murine retrovirusinduced depletion of T cells is mediated through activationinduced death by apoptosis," Journal of Virology, vol. 68, no. 4, pp. 2735-2740, 1994.
[14] K. Saha and P. K. Y. Wong, “T, not B, lymphocytes are required for immunodeficiency and paralysis induced by ts1, a mutant of Moloney murine leukemia virus-TB," Virology, vol. 183, no. 2, pp. 815-820, 1991.

[15] P. K. Y. Wong, G. Prasad, J. Hansen, and P. H. Yuen, “ts1, a mutant of Moloney murine leukemia virus-TB, causes both immunodeficiency and neurologic disorders in BALB/c mice," Virology, vol. 170, no. 2, pp. 450-459, 1989.

[16] P. H. Yuen, D. Malehorn, C. Knupp, and P. K. Y. Wong, "A 1.6-kilobase-pair fragment in the genome of the ts 1 mutant of Moloney murine leukemia virus TB that is associated with temperature sensitivity, nonprocessing of Pr80env, and paralytogenesis," Journal of Virology, vol. 54, pp. 364-373, 1985.

[17] C. G. Crispen Jr., Handbook of the Laboratory Mouse, Charles C Thomas, Springfield, Ill, USA, 1978.

[18] P. K. Y. Wong, E. Shikova, Y. C. Lin et al., "Murine leukemia virus induced central nervous system diseases," Leukemia, vol. 6, supplement 3, pp. 161S-165S, 1992.

[19] P. K. Y. Wong, "Moloney murine leukemia virus temperaturesensitive mutants: a model for retrovirus-induced neurologic disorders," Current Topics in Microbiology and Immunology, vol. 160, pp. 29-60, 1990.

[20] Y. Jiang, V. L. Scofield, M. Yan et al., "Retrovirus-induced oxidative stress with neuroimmunodegeneration is suppressed by antioxidant treatment with a refined monosodium alphaluminol (Galavit)," Journal of Virology, vol. 80, no. 9, pp. 45574569, 2006.

[21] P. K. Y. Wong, P. F. Szurek, E. Floyd, K. Saha, and B. R. Brooks, "Alteration from T- to B-cell tropism reduces thymic atrophy and cytocidal effects in thymocytes but not neurovirulence induced by ts1, a mutant of Moloney murine leukemia virus TB," Proceedings of the National Academy of Sciences of the United States of America, vol. 88, no. 20, pp. 8991-8995, 1991.

[22] K. Saha, D. Hollowell, and P. K. Y. Wong, "Mother-to-baby transfer of humoral immunity against retrovirus-induced neurologic disorders and immunodeficiency," Virology, vol. 198, no. 2, pp. 129-137, 1994.

[23] J. Chakraborty, S. Clark, H. Okonta, and J. Duggan, "A small animal model for mother-to-fetus transmission of ts 1 , a murine retrovirus," Viral Immunology, vol. 16, no. 2, pp. 191201, 2003.

[24] S. Fiore and M. L. Newell, "Preventing perinatal transmission of HIV-1 infection," Hospital Medicine, vol. 61, no. 5, pp. 315$318,2000$.

[25] D. T. Dunn, M. L. Newell, A. E. Ades, and C. S. Peckham, "Risk of human immunodeficiency virus type 1 transmission through breastfeeding," The Lancet, vol. 340, no. 8819, pp. 585-588, 1992.

[26] C. Wendo, "Most Ugandan HIV-positive mothers insist on breastfeeding," The Lancet, vol. 358, no. 9282, p. 649, 2001.

[27] A. Coutsoudis, K. Pillay, L. Kuhn, E. Spooner, W.-Y. Tsai, and H. M. Coovadia, "Method of feeding and transmission of HIV-1 from mothers to children by 15 months of age: prospective cohort study from Durban, South Africa," AIDS, vol. 15, no. 3, pp. 379-387, 2001.

[28] H. M. Coovadia, "Prevention and treatment of perinatal HIV1 infection in the developing world," Current Opinion in Infectious Diseases, vol. 13, no. 3, pp. 247-251, 2000.

[29] B. A. Richardson, G. C. John-Stewart, J. P. Hughes et al., "Breast-milk infectivity in human immunodeficiency virus 
type 1-infected mothers," Journal of Infectious Diseases, vol. 187, no. 5, pp. 736-740, 2003.

[30] Petra Study Team, "The Petra trial: the efficacy of three short-course regimens of zidovudine and lamivudine (3TC) in preventing early and late transmission of HIV-1 from mother to child in an African setting: a randomized, double-blind placebocontrolled trial, conducted in South Africa, Tanzania and Uganda," The Lancet, vol. 359, pp. 1178-1186, 2002.

[31] A. M. Herz, M. N. Robertson, J. B. Lynch et al., "Viral dynamics of early HIV infection in neonatal macaques after oral exposure to HIV-2 287 : an animal model with implications for maternal-neonatal HIV transmission," Journal of Medical Primatology, vol. 31, no. 1, pp. 29-39, 2002.

[32] R. M. Ruprecht, T. W. Baba, V. Liska et al., "Oral SIV, SHIV, and HIV type 1 infection," AIDS Research and Human Retroviruses, vol. 14, supplement 1, pp. S97-S103, 1998.

[33] R. M. Ruprecht, T. W. Baba, V. Liska et al., "Oral transmission of primate lentiviruses," Journal of Infectious Diseases, vol. 179, supplement 3, pp. S408-S412, 1999.

[34] N. C. Rollins, S. M. Filteau, A. Coutsoudis, and A. M. Tomkins, "Feeding mode, intestinal permeability, and neopterin excretion: a longitudinal study in infants of HIV-infected South African women," Journal of Acquired Immune Deficiency Syndromes, vol. 28, no. 2, pp. 132-139, 2001.

[35] R. Denison, "HIV and breastfeeding: what's a mother to do?" World, no. 126, pp. 5-7, 2001.

[36] WHO, HIV Transmission Through Breast-Feeding: A Review of Available Evidence, WHO, Geneve, Switzerland, 2004.

[37] P. K. Y. Wong, E. Floyd, and P. F. Szurek, "High susceptibility of $\mathrm{FVB} / \mathrm{N}$ mice to the paralytic disease induced by ts1, a mutant of Moloney murine leukemia virus TB," Virology, vol. 180, no. 1, pp. 365-371, 1991.

[38] K. Saha and P. K. Y. Wong, "Protective role of cytotoxic lymphocytes against murine leukemia virus-induced neurologic disease and immunodeficiency is enhanced by the presence of helper T cells," Virology, vol. 188, no. 2, pp. 921-925, 1992.

[39] V. Ellermann and O. Bang, "Experimentelle Leukämie bei Hühnern. Zentralbl. Bakteriol. Parasite," Infekt, vol. 46, pp. 595-609, 1908.

[40] P. Rous, "A sarcoma of the fowl transmissible by an agent separable from the tumor cells," Journal of Experimental Medicine, vol. 13, pp. 397-411, 1911.

[41] B. J. Poiesz, F. W. Ruscetti, A. F. Gazdar, P. A. Bunn, J. D. Minna, and R. C. Gallo, "Detection and isolation of type C retrovirus particles from fresh and cultured lymphocytes of a patient with cutaneous T-cell lymphoma," Proceedings of the National Academy of Sciences of the United States of America, vol. 77, no. 12, pp. 7415-7419, 1980.

[42] K. Barmak, E. Harhaj, C. Grant, T. Alefantis, and B. Wigdahl, "Human T cell leukemia virus type I-induced disease: pathways to cancer and neurodegeneration," Virology, vol. 308, no. 1, pp. 1-12, 2003.

[43] S. P. Goff, "Retroviridae: the retroviruses and their replication," in Field Virology, D. M. Knipe and P. M. Howley, Eds., vol. 2, Lippincott Williams \& Wilkins, Philadelphia, Pa, USA, 4th edition, 2000.

[44] C. Voisset, R. A. Weiss, and D. J. Griffiths, "Human RNA "rumor" viruses: the search for novel human retroviruses in chronic disease," Microbiology and Molecular Biology Reviews, vol. 72, no. 1, pp. 157-196, 2008.

[45] A. G. Uren, J. Kool, A. Berns, and M. van Lohuizen, "Retroviral insertional mutagenesis: past, present and future," Oncogene, vol. 24, no. 52, pp. 7656-7672, 2005.
[46] S. K. Shore, R. V. Tantravahi, and E. P. Reddy, "Transforming pathways activated by the $\mathrm{v}$-Abl tyrosine kinase," Oncogene, vol. 21, no. 56, pp. 8568-8576, 2002.

[47] K. T. Jeang and A. Gatignol, "Comparison of regulatory features among primate lentiviruses," Current Topics in Microbiology and Immunology, vol. 188, pp. 123-144, 1994.

[48] K. T. Jeang, "Retrovirology highlights a quarter century of HTLV-I research," Retrovirology, vol. 2, article 15, 2005.

[49] B. G. Herndier, B. T. Shiramizu, and M. S. McGrath, "AIDS associated non-Hodgkin's lymphomas represent a broad spectrum of monoclonal and polyclonal lymphoproliferative processes," Current Topics in Microbiology and Immunology, vol. 182, pp. 385-394, 1992.

[50] B. Shiramizu, B. G. Herndier, and M. S. McGrath, "Identification of a common clonal human immunodeficiency virus integration site in human immunodeficiency virus-associated lymphomas," Cancer Research, vol. 54, no. 8, pp. 2069-2072, 1994.

[51] L. Zamboni, J. Chakraborty, and D. M. Smith, "First cleavage division of the mouse zygot. An ultrastructural study," Biology of Reproduction, vol. 7, no. 2, pp. 170-193, 1972.

[52] Q. Wang, Y. Hirohashi, K. Furuuchi et al., "The centrosome in normal and transformed cells," DNA and Cell Biology, vol. 23, no. 8, pp. 475-489, 2004.

[53] E. A. Nigg, "Centrosome aberrations: cause or consequence of cancer progression?" Nature Reviews Cancer, vol. 2, no. 11, pp. 815-825, 2002.

[54] H. Zhou, J. Kuang, L. Zhong et al., “Tumour amplified kinase STK15/BTAK induces centrosome amplification, aneuploidy and transformation," Nature Genetics, vol. 20, no. 2, pp. 189193, 1998.

[55] H. Fan, S. C. Kim, C. O. Chima et al., "Epstein-Barr viral load as a marker of lymphoma in AIDS patients," Journal of Medical Virology, vol. 75, no. 1, pp. 59-69, 2005.

[56] J. Li, H. Shen, K. L. Himmel et al., "Leukaemia disease genes: large-scale cloning and pathway predictions," Nature Genetics, vol. 23, no. 3, pp. 348-353, 1999.

[57] X. Liao, A. M. Buchberg, N. A. Jenkins, and N. G. Copeland, "Evi-5, a common site of retroviral integration in AKXD Tcell lymphomas, maps near Gfi-1 on mouse chromosome 5," Journal of Virology, vol. 69, no. 11, pp. 7132-7137, 1995.

[58] B. Scheijen, J. Jonkers, D. Acton, and A. Berns, "Characterization pal-1, a common proviral insertion site in murine leukemia virus-induced lymphomas of c-myc and Pim-1 transgenic mice," Journal of Virology, vol. 71, no. 1, pp. 9-16, 1997.

[59] M. S. Shin, T. N. Fredrickson, J. W. Hartley, T. Suzuki, K. Akagi, and H. C. Morse III, "High-throughput retroviral tagging for identification of genes involved in initiation and progression of mouse splenic marginal zone lymphomas," Cancer Research, vol. 66, no. 6, pp. 4419-4427, 2006.

[60] F. Gergely, C. Karlsson, I. Still, J. Cowell, J. Kilmartin, and J. W. Raff, "The TACC domain identifies a family of centrosomal proteins that can interact with microtubules," Proceedings of the National Academy of Sciences of the United States of America, vol. 97, no. 26, pp. 14352-14357, 2000.

[61] C. K. Jung, J. H. Jung, G. S. Park, A. Lee, C. S. Kang, and K. Y. Lee, "Expression of transforming acidic coiled-coil containing protein 3 is a novel independent prognostic marker in nonsmall cell lung cancer," Pathology International, vol. 56, no. 9, pp. 503-509, 2006. 
[62] B. Lauffart, M. M. Vaughan, R. Eddy et al., "Aberrations of TACC1 and TACC3 are associated with ovarian cancer," BMC Women's Health, vol. 5, article 8, 2005.

[63] L. Schneider, F. Essmann, A. Kletke et al., "The transforming acidic coiled coil 3 protein is essential for spindle-dependent chromosome alignment and mitotic survival," Journal of Biological Chemistry, vol. 282, no. 40, pp. 29273-29283, 2007.

[64] R. P. Piekorz, A. Hoffmeyer, C. D. Duntsch et al., "The centrosomal protein TACC3 is essential for hematopoietic stem cell function and genetically interfaces with $p 53$-regulated apoptosis," EMBO Journal, vol. 21, no. 4, pp. 653-664, 2002.

[65] P. J. LeRoy, J. J. Hunter, K. M. Hoar et al., "Localization of human TACC3 to mitotic spindles is mediated by phosphorylation on Ser558 by aurora A: a novel pharmacodynamic method for measuring aurora A activity," Cancer Research, vol. 67, no. 5362, pp. 5362-5370, 2007.

[66] R. Crane, B. Gadea, L. Littlepage, H. Wu, and J. V. Ruderman, "Aurora A, meiosis and mitosis," Biology of the Cell, vol. 96, no. 3, pp. 215-229, 2004.

[67] C. A. Maxwell, J. J. Keats, A. R. Belch, L. M. Pilarski, and T. Reiman, "Receptor for hyaluronan-mediated motility correlates with centrosome abnormalities in multiple myeloma and maintains mitotic integrity," Cancer Research, vol. 65, no. 3, pp. 850-860, 2005.

[68] D. Mori, Y. Yano, K. Toyo-Oka et al., "NDEL1 phosphorylation by aurora-A kinase is essential for centrosomal maturation, separation, and TACC3 recruitment," Molecular and Cellular Biology, vol. 27, no. 1, pp. 352-367, 2007. 

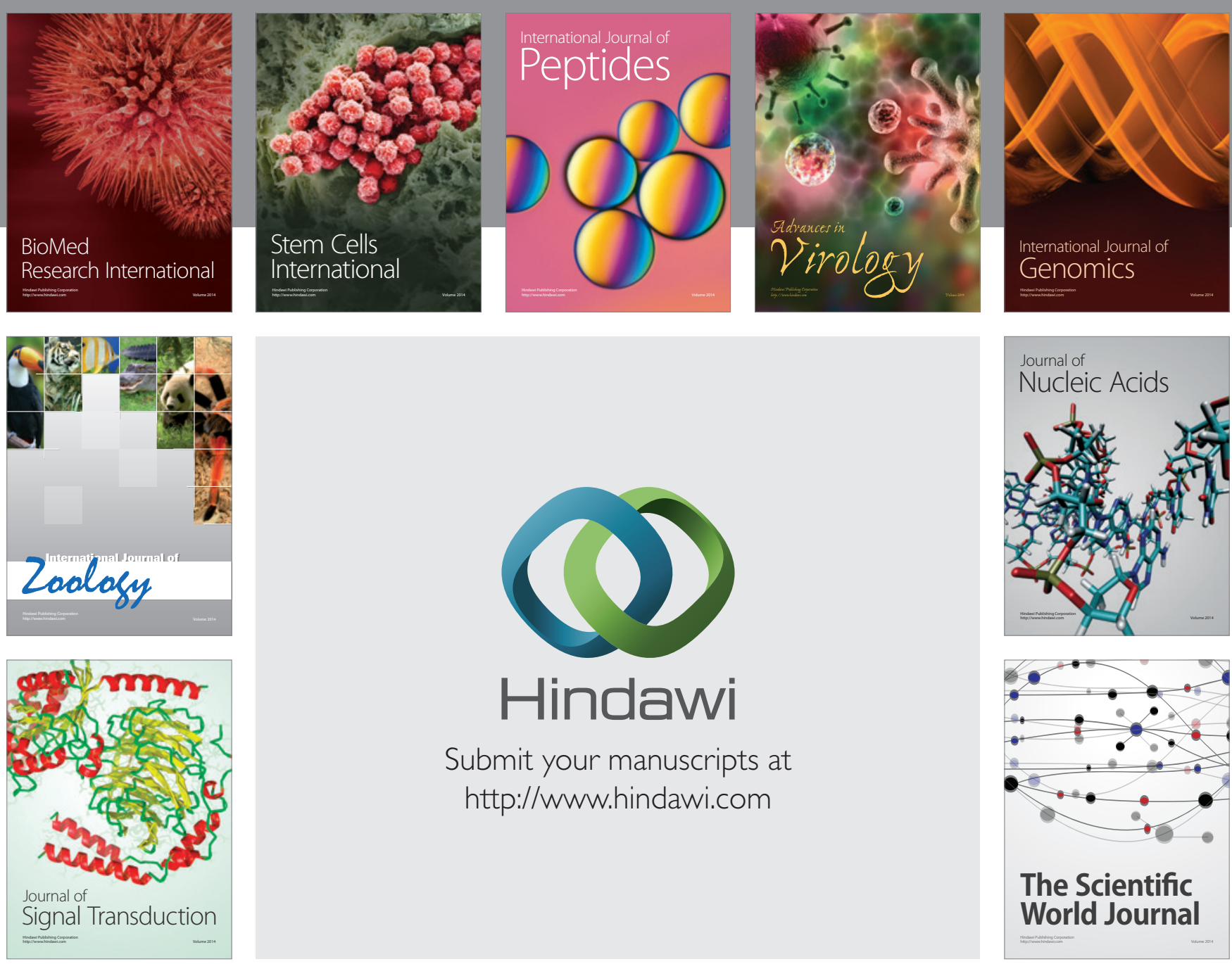

Submit your manuscripts at

http://www.hindawi.com
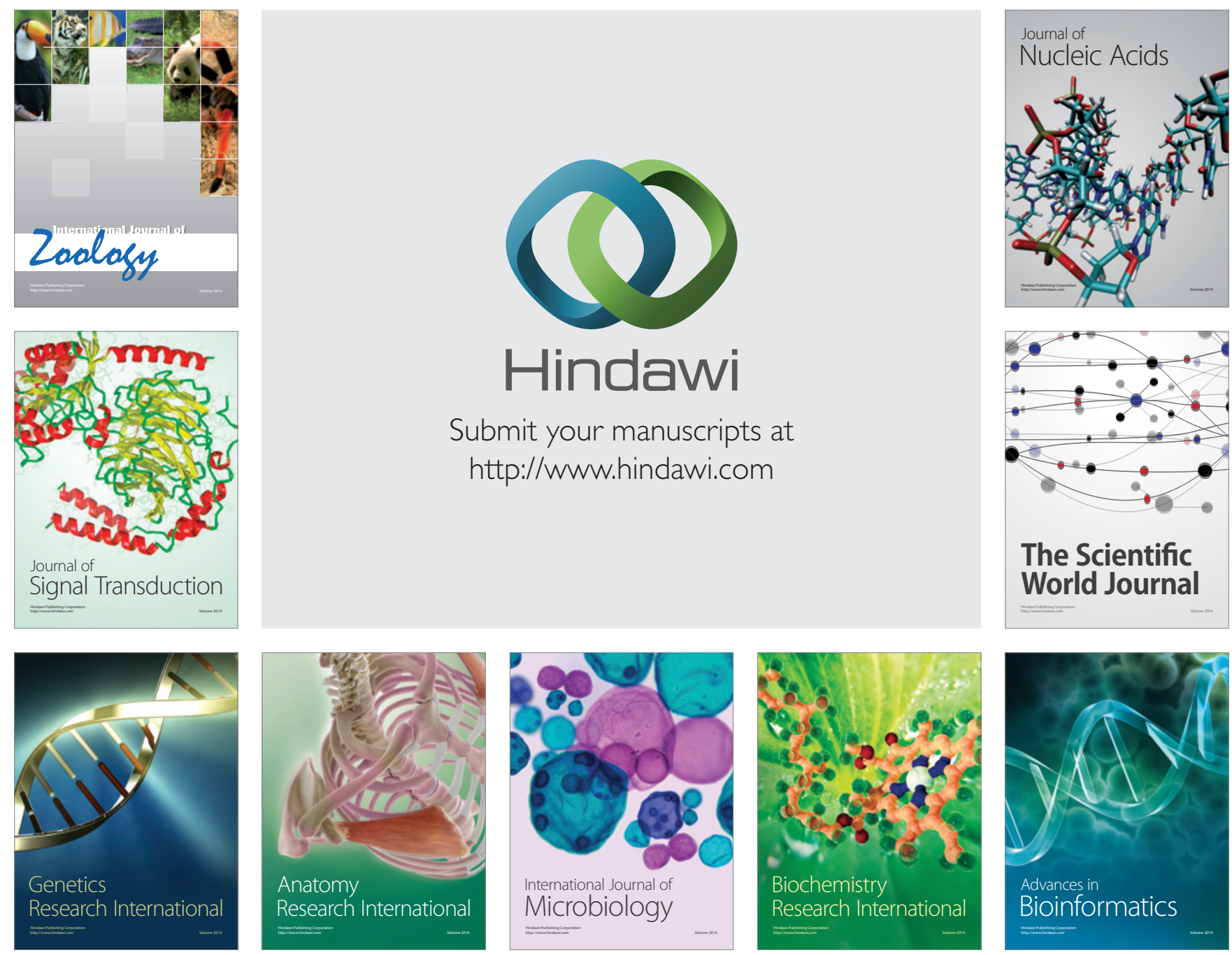

The Scientific World Journal
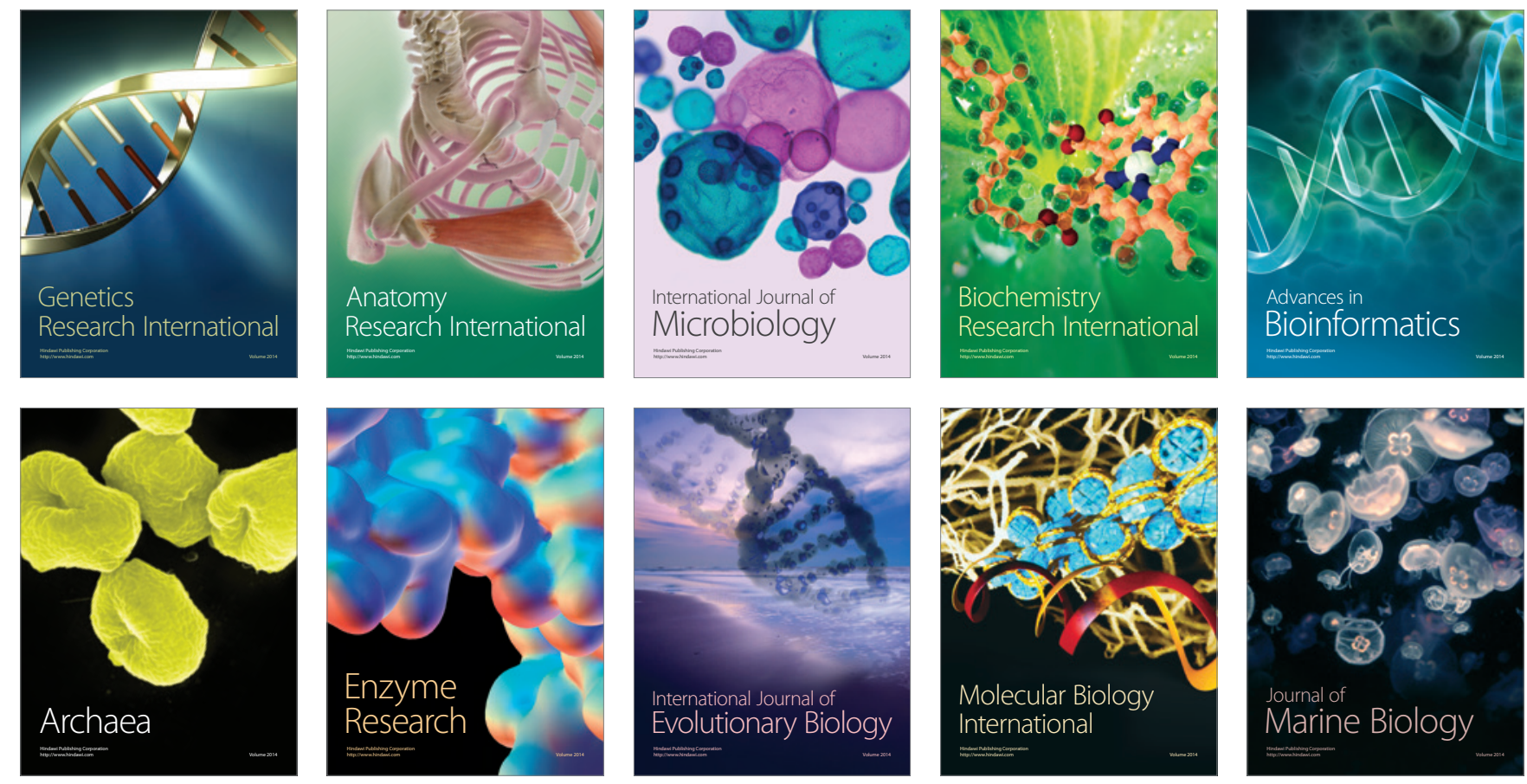